\title{
ON MINIMAL NON-SCATTERING SOLUTION FOR FOCUSING MASS-SUBCRITICAL NONLINEAR SCHRÖDINGER EQUATION
}

\author{
SATOSHI MASAKI \\ Laboratory of Mathematics, Institute of Engineering, Hiroshima University, \\ Higashihiroshima Hirhosima, 739-8527, Japan \\ masaki@amath.hiroshima-u.ac.jp

\begin{abstract}
We consider time global behavior of solutions to the focusing mass-subcritical NLS equation in weighted $L^{2}$ space. We prove that there exists a threshold solution such that (i) it does not scatter; (ii) with respect to a certain scale-invariant quantity, this solution attains minimum value in all non-scattering solutions. In the mass-critical case, it is known that ground states are this kind of threshold solution. However, in our case, it turns out that the above threshold solution is not a standing-wave solution.
\end{abstract}

\section{INTRODUCTION}

In this article, we study the initial value problem of the following nonlinear Schrödinger equation

$$
\left\{\begin{array}{l}
i \partial_{t} u+\Delta u=\lambda|u|^{p-1} u, \quad(t, x) \in \mathbb{R}^{1+N} \\
u(0)=u_{0} \in \mathcal{F} H^{1}
\end{array}\right.
$$

where $N \geqslant 1$ and $\lambda=-1$. The initial data $u_{0}$ is taken from weighted $L^{2}$ space

$$
\mathcal{F} H^{1}=\mathcal{F} H^{1}\left(\mathbb{R}^{N}\right):=\left\{f \in L^{2}\left(\mathbb{R}^{N}\right) \mid x f \in L^{2}\left(\mathbb{R}^{N}\right)\right\}
$$

with norm

$$
\|f\|_{\mathcal{F} H^{1}}^{2}=\|f\|_{L^{2}}^{2}+\|x f\|_{L^{2}}^{2} .
$$

We treat short range, mass-subcritical, and $\mathcal{F} \dot{H}^{1}$-supercritical case;

$$
\max \left(1+\frac{2}{N}, 1+\frac{4}{N+2}\right)<p<1+\frac{4}{N} .
$$

It is known that the initial value problem (NLS) is globally well-posed in $\mathcal{F} H^{1}$ (See, [19] and reference therein). More precisely, for any $u_{0} \in \mathcal{F} H^{1}$ there exists a solution $u(t)$ such that $e^{-i t \Delta} u(t) \in C\left(\mathbb{R}, \mathcal{F} H^{1}\right)$ and conserves mass

$$
M[u(t)]:=\|u(t)\|_{L^{2}}^{2} .
$$

Let us refer a solution in this class as an $\mathcal{F} H^{1}$-solution in what follows. Remark that, by conservation of mass, $\mathcal{F} H^{1}$-solutions do not blow up in finite time. The equation (NLS) has the following scaling; if $u(t, x)$ is a solution to (NLS) then for all $\omega>0$

$$
u_{\omega}(t, x)=\omega^{\frac{2}{p-2}} u\left(\omega^{2} t, \omega x\right)
$$


is also a solution to (NLS). Hidano proved existence of small radial solutions of (LS) for data in a scale-invariant homogeneous Sobolev space $\dot{H}^{-s}$ in [12.

Our concern is global behavior of $\mathcal{F} H^{1}$-solutions of ( $(\overline{N L S})$. In principle, behavior of a solution is governed by a balance between dispersive effect by free Schrödinger evolution and nonlinear interaction. If the linear dispersive effect becomes dominant for large time, then a solution asymptotically behaves as a free solution (scattering). For the precise definition of scattering, see Definition 1.1, below. In the case where $1+\frac{2}{N}<p \leqslant 1+\frac{4}{N}$ and $p \geqslant 1+\frac{4}{N+2}$, it is known that an $\mathcal{F} H^{1}$-solution scatters in $\mathcal{F} H^{1}$ for both time directions, provided its initial value is sufficiently small in $\mathcal{F} H^{1}$ sense (see [5, 10, 19]). Notice that smallness of datum gives that of corresponding solution, which is closely related to weakness of nonlinear effect relative to linear effect. We also remark that the assumption $p>1+\frac{2}{N}$ is essential for considering scattering phenomenon since it is known that if $p \leqslant 1+\frac{2}{N}$ then any non-trivial solution does not scatter (see [3, 20]). In the defocusing case $\lambda=+1$, it is further known that, for any data $u_{0} \in \mathcal{F} H^{1}$, the solution $u$ scatters for both positive and negative time as long as $1+\frac{4}{N}>p \geqslant p_{\text {St }}$, where

$$
p_{\mathrm{St}}:=1+\frac{2-N+\sqrt{N^{2}+12 N+4}}{2 N},
$$

see [21, 5, 19]. The lower bound $p_{\mathrm{St}}$, which is sometimes referred to as a Strauss exponent, is a root of $N p^{2}-(N+2) p-2=0$. Remark that $p_{\mathrm{St}}>\max \left(1+\frac{2}{N}, 1+\frac{4}{N+2}\right)$ for all $N \geqslant 1$. However, the situation differs in the focusing case $\lambda=-1$ because the nonlinear interaction conflicts with linear dispersive effect. When datum is not small, that is, when nonlinear effect is not weak, a solution does not necessarily scatter. A typical example of non-scattering solution is a standing wave solution $e^{i \omega^{2} t} \varphi_{\omega}(x)$, where $\omega>0$ and $\varphi_{\omega}(x)=\omega^{\frac{2}{p-1}} \psi(\omega x)$ with

$$
-\Delta \psi+\psi=|\psi|^{p-1} \psi
$$

There exists a unique positive radial solution $Q(x)$ to (1.3) called the ground state, provided $1<p<1+\frac{4}{N-2}(1<p<\infty$ if $N=1,2)$, see [4] and references therein. It is characterized as the solution minimizing the energy

$$
E[u(t)]:=\frac{1}{2}\|\nabla u(t)\|_{L^{2}}^{2}-\frac{1}{p+1}\|u(t)\|_{L^{p+1}}^{p+1}
$$

among all nontrivial $H^{1}$-solutions.

In this article, we consider the transition between scattering and nonscattering. First main result is existence of a threshold solution (Theorem 1.3). More precisely, we show the following: Let us introduce

$$
\ell(f):=\|f\|_{L^{2}}^{\frac{N+2}{2}-\frac{2}{p-1}}\|x f\|_{L^{2}}^{\frac{2}{p-1}-\frac{N}{2}},
$$

which is well defined for $f \in \mathcal{F} H^{1}$. If $p>p_{\mathrm{St}}$ then there exists a special $\mathcal{F} H^{1}$-solution $u_{\mathrm{c}}(t)$ such that (i) it does not scatter for positive time; (ii) $u_{\mathrm{c}}(t)$ attains minimum value of $\ell(u(0))$ among all non-scattering solutions. From these respects, we refer $u_{\mathrm{c}}(t)$ to as a minimal non-scattering solution. The second assertion gives the following sharp criteria for scattering; if an 
$\mathcal{F} H^{1}$-solution $u(t)$ of (NLS) satisfies $\ell(u(0))<\ell\left(u_{\mathrm{c}}(0)\right)$ then the solution $u(t)$ scatters for both positive and negative time. Notice that $\ell(u(0))$ is invariant under the scaling (1.1).

Our result is similar to the one in the mass-critical case $p=1+\frac{4}{N}$. Dodson shows in [6] that if an $L^{2}$-solution $u(t)$ satisfies $\|u(0)\|_{L^{2}}<\|Q\|_{L^{2}}$ then the solution exists globally in time and scatters in $L^{2}$ for both positive and negative time. In our terminology, this result can be rephrased as "the ground states are the minimal non-scattering solutions." However, in the mass-subcritical case, it turns out that the minimal non-scattering solution is not neither a ground state nor any other standing wave solutions, which is our second result (Theorem 1.7).

In the energy-critical case $p=1+\frac{4}{N-2}(N \geqslant 3)$, global behavior of solutions of which initial data belongs to a scale-invariant set

$$
K=\left\{u_{0} \in \dot{H}^{1} \mid E\left[u_{0}\right]<E[W]\right\}
$$

is considered, where $W=\left(1+\frac{|x|^{2}}{N(N-2)}\right)^{-\frac{N-2}{2}}$ is a solution to an elliptic equation $\Delta W+|W|^{\frac{4}{d-2}} W=0$. It is shown that $K$ is written as $K=S \cup B$, where $S$ and $B$ are invariant under the NLS flow and satisfy $S \cap B=\emptyset$ and $S \ni 0$. If $u_{0} \in S$ then a solution of (NLS) with $\left.u\right|_{t=0}=u_{0}$ scatters for both time directions, and if $u_{0} \in B$ and if either $u_{0} \in \mathcal{F} \dot{H}^{1}$ or $u_{0} \in L^{2}$ is radial then the solution blows up in finite time. This is first given by Kenig and Merle [15] for $3 \leqslant N \leqslant 5$ under radial assumption, and is extended by Killip and Visan [17] to non-radial $N \geqslant 5$ case. In the energy-subcritical and mass-supercritical case $1+\frac{4}{N}<p<1+\frac{4}{N-2}\left(1+\frac{4}{N}<p<\infty\right.$ if $\left.N=1,2\right)$, a similar classification result is obtained for $H^{1}$-solutions belonging to

$$
\begin{aligned}
L=\left\{u_{0} \in H^{1} \mid\right. & M\left[u_{0}\right]^{\frac{2}{p-1}-\frac{N-2}{2}} E\left[u_{0}\right]^{\frac{N}{2}-\frac{2}{p-1}} \\
& \left.<M[Q]^{\frac{2}{p-1}-\frac{N-2}{2}} E[Q]^{\frac{N}{2}-\frac{2}{p-1}}\right\},
\end{aligned}
$$

see [1, 13] (see also [7, 8] for scattering part).

In this article, we do not work with $H^{1}$-solutions in the following two respects. First, one can find an arbitrarily small (in $H^{1}$ sense) non-scattering solution in the family of ground state solutions. Hence, it seems difficult to yield any classification similar to those in previous results. Second, a sufficient condition for scattering is boundedness of a scale-invariant spacetime norm of the form $\|u\|_{L_{t}^{\rho}\left(\mathbb{R}, L_{x}^{\gamma}\left(\mathbb{R}^{N}\right)\right)}$. In the mass-subcritical case, this quantity is not necessarily bounded for $H^{1}$-solutions. An answer to these problems is to work with $\mathcal{F} H^{1}$-solutions. However, to do so, we must recast a concentration-compactness argument, which is first carried out in 15 for $\dot{H}^{1}$ solutions and later extended to $H^{1}$-solutions, in a form adapted to $\mathcal{F} H^{1}$-solutions. This adaption is the main technical issue on this paper. It then turns out that there is a difference in a so-called profile decomposition lemma. More precisely, a bounded sequences in $\mathcal{F} H^{1}$ are decomposed into a simpler form than those in the known decompositions of $H^{1}$ - or $\dot{H}^{1}$-bounded sequences.

Main results. We now state our results precisely. 
Definition 1.1. Let $X$ be a Banach space. Let u be a solution to (NLS) such that $e^{-i t \Delta} u(t) \in X$ for any $t \in \mathbb{R}$. We say $u$ scatters in $X$ for positive time (resp. negative time) if $\lim _{t \rightarrow \infty} e^{-i t \Delta} u(t)$ exists (resp. $\lim _{t \rightarrow-\infty} e^{-i t \Delta} u(t)$ exists) in $X$.

Definition 1.2. We define $S_{+}, S_{-} \subset \mathcal{F} H^{1}$ as follows:

$$
\begin{aligned}
& S_{+}=\left\{\begin{array}{l|l}
u_{0} \in \mathcal{F} H^{1} & \begin{array}{l}
\text { solution } u(t) \text { of (ㄴS }) \text { with }\left.u\right|_{t=0}=u_{0} \\
\text { scatters in } \mathcal{F} H^{1} \text { for positive time }
\end{array}
\end{array}\right\}, \\
& S_{-}=\left\{\begin{array}{l|l}
u_{0} \in \mathcal{F} H^{1} & \begin{array}{l}
\text { solution } u(t) \text { of (LS) with }\left.u\right|_{t=0}=u_{0} \\
\text { scatters in } \mathcal{F} H^{1} \text { for negative time }
\end{array}
\end{array}\right\} .
\end{aligned}
$$

Further, we define a scattering set $S$ as $S=S_{+} \cap S_{-}$.

The first main result of this article is

Theorem 1.3. Suppose $\lambda=-1$ and $p \in\left(p_{\mathrm{St}}, 1+\frac{4}{N}\right)$, where $p_{\mathrm{St}}$ is given in (1.2). Then, there exists $u_{0, c} \in \mathcal{F} H^{1}$ satisfying the following two properties:

(1) $u_{0, \mathrm{c}} \notin S_{+} \quad$ (non-scattering);

(2) $\ell\left(u_{0, \mathrm{c}}\right)=\inf _{u_{0} \in \mathcal{F} H^{1} \backslash S} \ell\left(u_{0}\right) \quad$ (minimality).

The solution $u_{\mathrm{c}}$ with $\left.u_{\mathrm{c}}\right|_{t=0}=u_{0, \mathrm{c}}$ is the minimal non-scattering solution.

Remark 1.4. The property (2) of Theorem 1.3 implies that if $u_{0} \in \mathcal{F} H^{1}$ satisfies $\ell\left(u_{0}\right)<\ell\left(u_{0, \mathrm{c}}\right)$ then $u_{0} \in S$. This criteria is sharp in view of the property (1).

Remark 1.5. If $u(t)$ is a solution then $\bar{u}(-t)$ is also a solution. This implies $\overline{u_{0}} \in S_{-}$if and only if $u_{0} \in S_{+}$. Then, we see that $v_{0, c}:=\overline{u_{0, c}}$ satisfies $v_{0, c} \notin S_{-}$and the property (2) of Theorem 1.3 since $\ell(u(0))=\ell(\bar{u}(0))$.

Remark 1.6. We do not know whether $u_{0, \mathrm{c}} \in S_{-}$or not.

Our proof of Theorem 1.3 is based on a concentration-compactness method introduced in [15]. In the mass-critical and -supercritical cases, one makes use of a rigidity theorem together with concentration-compactness argument to yield a contradiction from an assumption that a threshold solution is smaller than the ground state in a suitable sense. However, in our case, no contradiction can be derived from the assumption $\ell\left(u_{0, c}\right)<\ell(Q)$ because this assertion is actually true.

Theorem 1.7. For any standing-wave solution $e^{i \omega^{2} t} \varphi_{\omega}(x)$, it holds that $\ell\left(\varphi_{\omega}\right)>\inf _{u_{0} \in \mathcal{F} H^{1} \backslash S} \ell\left(u_{0}\right)$. In particular, the minimal non-scattering solution $u_{\mathrm{c}}$ given in Theorem 1.3 is not a standing-wave solution.

Remark 1.8. More generally, if $\psi \in H^{1} \cap \mathcal{F} H^{1}$ has negative energy then $\ell(\psi)>\inf _{u_{0} \in \mathcal{F} H^{1} \backslash S} \ell\left(u_{0}\right)$, in fact (see Theorem 3.2). Let us note that, in the case $1+\frac{4}{N}<p<1+\frac{4}{N-2}\left(1+\frac{4}{N}<p<\infty\right.$ if $\left.N=1,2\right)$, the same assumption on $u_{0}$ yields finite time blowup [11].

The rest of the article is organized as follows. In section 2, we briefly recall some basic facts and well-posedness result in $\mathcal{F} H^{1}$, and then give a necessary and sufficient condition for scattering. Section 3 is devoted to 
the proof of Theorem 1.7. Then, we turn to the proof of Theorem 1.3. In Sections 4 and 5, several tools for a concentration-compactness method is established in the framework of weighted $L^{2}$ space. We first prove a socalled long time perturbation theory for $\mathcal{F} H^{1}$-solutions in Section 4. Several consequent results such as small data scattering or oscillating data scattering are also shown there. The latter says that a solution scatters for both time directions if its initial data is sufficiently oscillating, which is specific to the mass-subcritical case and the key for the proof of the profile decomposition lemma for bounded sequences in $\mathcal{F} H^{1}$, considered in Section 5. Finally, we complete the proof of Theorem 1.3 in Section 6 .

\section{NECESSARY AND SUFFICIENT CONDITION FOR SCATTERING IN $\mathcal{F} H^{1}$}

Let us begin with introduction of notations. We write $2^{*}=\frac{2 N}{N-2}$ for $N \geqslant 3$. Let $p^{\prime}=\frac{p}{p-1}$ and $\delta(r):=N\left(\frac{1}{2}-\frac{1}{r}\right)$. We say a pair $(q, r)$ is admissible if $r \in\left[2,2^{*}\right](r \in[2, \infty]$ if $N=1, r \in[2, \infty)$ if $N=2)$ and $q \delta(r)=2$. For any admissible pairs $(q, r),\left(q_{1}, r_{1}\right)$, and $\left(q_{2}, r_{2}\right)$, Strichartz' estimates hold. There exists a constant $C$ such that

$$
\left\|e^{i t \Delta} f\right\|_{L^{q}\left(I, L^{r}\right)} \leqslant C\|f\|_{L^{2}}
$$

for all interval $I \subset \mathbb{R}$ and $f \in L^{2}$. There also exists a constant $C$ such that

$$
\left\|\int_{t_{0}}^{t} e^{i(t-s) \Delta} g(s) d s\right\|_{L^{q_{1}\left(I, L^{r_{1}}\right)}} \leqslant C\|g\|_{L^{q_{2}^{\prime}\left(I, L^{r_{2}^{\prime}}\right)}}
$$

for any $t_{0} \in I \subset \mathbb{R}$ and $g \in L^{q_{2}^{\prime}}\left(I, L_{2}^{r_{2}^{\prime}}\right)$.

Throughout this paper, we use the following notation.

$$
\begin{array}{ll}
\rho=\frac{2\left(p^{2}-1\right)}{4-(N-2)(p-1)}, & \gamma=p+1, \\
\widetilde{\rho}=\frac{2\left(p^{2}-1\right)}{N p^{2}-(N+2) p-2}, & \widetilde{\gamma}=p+1 .
\end{array}
$$

There are well defined for $p \in\left(p_{\mathrm{St}}, 1+\frac{4}{N}\right)$. Note that the $L_{t}^{\rho}\left(\mathbb{R}, L_{x}^{\gamma}\left(\mathbb{R}^{N}\right)\right)$ norm is invariant under the scaling (1.1). Moreover, the relations

$$
\rho=p \widetilde{\rho}^{\prime}, \quad \gamma=p \widetilde{\gamma}^{\prime}
$$

hold. We also remark that $(\rho, \gamma)$ is not an admissible pair since

$$
2>\rho \delta(\gamma)
$$

follows by definition. We have non-admissible Strichartz' estimate holds for $(\rho, \gamma, \widetilde{\rho}, \widetilde{\gamma})([14]) ;$ there exists a constant $C>0$ such that, for any interval $I \subset \mathbb{R}$ and $t_{0} \in I$, we have

$$
\left\|\int_{t_{0}}^{t} e^{i(t-s) \Delta} g(s) d s\right\|_{L^{\rho}\left(I, L^{\gamma}\right)} \leqslant C\|g\|_{L^{\tilde{\rho}^{\prime}\left(I, L \tilde{\gamma}^{\prime}\right)}}
$$

for all $g \in L^{\widetilde{q}^{\prime}}\left(I, L^{\widetilde{r}^{\prime}}\right)$ For more precise conditions of this estimate, see [9, 18, 23. One verifies that if $p>p_{\mathrm{St}}$ then

$$
\rho \delta(\gamma)>1, \quad \tilde{\rho} \delta(\widetilde{\gamma})>1
$$

holds. 
Next, let us collect some facts on $J(t):=x+2 i t \nabla$. We have

$$
\left[i \partial_{t}+\Delta, J(t)\right]=0
$$

and $J(t)$ is written as

$$
J(t)=e^{i \frac{|x|^{2}}{2 t}} 2 i t \nabla e^{-i \frac{|x|^{2}}{2 t}}=e^{i t \Delta} x e^{-i t \Delta} .
$$

Lemma 2.1. If $r \in\left[2,2^{*}\right](r \in[2, \infty]$ if $N=1, r \in[2, \infty)$ if $N=2)$, then

$$
\|f\|_{L^{\gamma}} \leqslant C|t|^{-\delta(r)}\|f\|_{L^{2}}^{1-\delta(r)}\|J(t) f\|_{L^{2}}^{\delta(r)}
$$

for any $t \neq 0$. Further, there exists a constant $C>0$ such that

$$
\left\|e^{i t \Delta} f\right\|_{L_{t}^{\rho}\left(\mathbb{R}, L_{x}^{\gamma}\left(\mathbb{R}^{N}\right)\right)} \leqslant C \ell(f)
$$

holds for $f \in \mathcal{F} H^{1}$.

Proof. By the Sobolev embedding and the first identity of (2.6), we immediately obtain

$$
\begin{aligned}
\|f\|_{L^{r}} & =\left\|e^{-i \frac{|x|^{2}}{2 t}} f\right\|_{L^{r}} \leqslant C\left\|e^{-i \frac{|x|^{2}}{2 t}} f\right\|_{L^{2}}^{1-\delta(r)}\left\|\nabla e^{-i \frac{|x|^{2}}{2 t}} f\right\|_{L^{2}}^{\delta(r)} \\
& \leqslant C|t|^{-\delta(r)}\|f\|_{L^{2}}^{1-\delta(r)}\|J(t) f\|_{L^{2}}^{\delta(r)} .
\end{aligned}
$$

Let us proceed to the proof of second inequality. We may assume $f \neq 0$, otherwise the result is obvious. Let $T_{f}=\|x f\|_{L^{2}}^{2} /\|f\|_{L^{2}}^{2}>0$. Since $e^{i t \Delta}$ is unitary on $L^{2}$, we see from the identity just shown and the second identity of (2.6) that

$$
\begin{aligned}
\left\|e^{i t \Delta} f\right\|_{L^{\gamma}} & \leqslant C|t|^{-\delta(\gamma)}\left\|e^{i t \Delta} f\right\|_{L^{2}}^{1-\delta(\gamma)}\left\|J(t) e^{i t \Delta} f\right\|_{L^{2}}^{\delta(\gamma)} \\
& =C|t|^{-\delta(\gamma)}\|f\|_{L^{2}}^{1-\delta(\gamma)}\|x f\|_{L^{2}}^{\delta(\gamma)} .
\end{aligned}
$$

Since $\rho \delta(\gamma)>1$ as noticed in (2.5),

$$
\begin{aligned}
\left\|e^{i t \Delta} f\right\|_{L^{\rho}\left(\mathbb{R} \backslash\left[-T_{f}, T_{f}\right], L^{\gamma}\right)} & \leqslant C T_{f}^{\frac{1}{\rho}-\delta(\gamma)}\|f\|_{L^{2}}^{1-\delta(\gamma)}\|x f\|_{L^{2}}^{\delta(\gamma)} \\
& =C\|f\|_{L^{2}}^{1-\left(\frac{2}{\rho}-\delta(\gamma)\right)}\|x f\|_{L^{2}}^{\frac{2}{\rho}-\delta(\gamma)}=C \ell(f) .
\end{aligned}
$$

On the other hand, by Hödler's inequality and Strichartz' estimate,

$$
\begin{aligned}
\left\|e^{i t \Delta} f\right\|_{L^{\rho}\left(\left[-T_{f}, T_{f}\right], L^{\gamma}\right)} & \leqslant C T_{f}^{\frac{1}{\rho}-\frac{\delta(\gamma)}{2}}\left\|e^{i t \Delta} f\right\|_{L^{\frac{2}{\delta(\gamma)}\left(\left[-T_{f}, T_{f}\right], L^{\gamma}\right)}} \\
& \leqslant C T_{f}^{\frac{1}{\rho}-\frac{\delta(\gamma)}{2}}\|f\|_{L^{2}}=C \ell(f),
\end{aligned}
$$

which completes the proof.

We summarize basic well-posedness result in $\mathcal{F} H^{1}$ and sufficient condition for scattering in a form suitable for our later use. This is a consequence of [10, 19]. However, we give a brief proof for readers' convenience.

Proposition 2.2 (Global well-posedness, sufficient condition for scattering). Suppose $1<p<1+4 / N$. Then, equation (NLS) is globally well-posed in $L^{2}$. Moreover, the solution scatters in $L^{2}$ for positive time if

$$
\|u\|_{L^{\rho}\left((0, \infty), L^{\gamma}\right)}<\infty .
$$

Furthermore, the following hold. 
- If $u_{0} \in \mathcal{F} H^{1}$ (resp. $\left.u_{0} \in H^{1}\right)$ then the solution satisfies $e^{-i t \Delta} u(t) \in$ $C\left(\mathbb{R}, \mathcal{F} H^{1}\right)\left(\right.$ resp. $\left.u(t) \in C\left(\mathbb{R}, H^{1}\right)\right)$.

- If $u_{0} \in \mathcal{F} H^{1}$ (resp. $\left.u_{0} \in H^{1}\right)$ and if (2.7) is satisfied then $e^{-i t \Delta} u(t)$ converges in $\mathcal{F} H^{1}$ (resp. in $H^{1}$ ) as $t \rightarrow \infty$.

Remark 2.3. The condition (2.7) is never satisfied with a non-trivial solution, provided $p \leqslant 1+\frac{2}{N}$. When $p<p_{\mathrm{St}}, \mathcal{F} H^{1}$-solutions of the free Scrhödinger equation do not satisfy (2.7) in general.

Proof. Global well-posedness in $L^{2}$ is well known (see [22]). Namely, for all $u_{0} \in L^{2}$, there exists a unique global solution $u(t)$ belonging to $C\left(\mathbb{R}, L^{2}\right) \cap$ $L_{\text {loc }}^{a}\left(\mathbb{R}, L^{b}\left(\mathbb{R}^{N}\right)\right)$ for any admissible pair $(a, b)$. Note that $u \in L_{\text {loc }}^{\rho}\left(\mathbb{R}, L^{\gamma}\left(\mathbb{R}^{N}\right)\right)$ follows from $\rho \delta(\gamma)<2$.

Let us prove the scattering. Assume (2.7). Take an admissible pair $(q, r)$ so that

$$
1-\frac{2}{r}=\frac{p-1}{\gamma} \text {. }
$$

This is possible since $\delta(r)=\frac{N(p-1)}{2(p+1)} \in(0,1)$. A use of Strichartz's estimate gives us

$$
\|u\|_{L^{q}\left(I, L^{r}\right)} \leqslant C\left\|u\left(t_{0}\right)\right\|_{L^{2}}+C\left\||u|^{p-1} u\right\|_{L^{q^{\prime}\left(I, L^{r^{\prime}}\right)}} .
$$

for any $t_{0} \in I \subset \mathbb{R}$. By conservation of mass and Hölder inequality,

$$
\|u\|_{L^{q}\left(I, L^{r}\right)} \leqslant C\left\|u_{0}\right\|_{L^{2}}+C\|u\|_{L^{\rho}\left(I, L^{\gamma}\right)}^{p-1}\|u\|_{L^{q}\left(I, L^{r}\right)} .
$$

Since $\|u\|_{L^{\rho}\left(\mathbb{R}_{+}, L^{\gamma}\right)}<\infty$ by assumption, for sufficiently large $\tilde{T}$, we obtain

$$
\|u\|_{L^{q}\left(\left([\tilde{T}, T], L^{r}\right)\right.} \leqslant C\left\|u_{0}\right\|_{L^{2}}+\frac{1}{2}\|u\|_{L^{q}\left(\left([\tilde{T}, T], L^{r}\right)\right.}
$$

for any $T>\widetilde{T}$, which shows $\|u\|_{L^{q}\left(\left([\tilde{T}, T], L^{r}\right)\right.} \leqslant 2 C\left\|u_{0}\right\|_{L^{2}}$. Since $T$ is arbitrary, $\|u\|_{L^{q}\left(\left([\tilde{T}, \infty), L^{r}\right)\right.}<\infty$. Applying Strichartz' estimate again, we see that $u \in L^{a}\left([0, \infty), L^{b}\right)$ for all admissible pair $(a, b)$. One then deduces that

$$
\psi_{+}:=u_{0}-i \lambda \int_{0}^{\infty} e^{-i s \Delta}\left(|u|^{p-1} u\right)(s) d s
$$

is well-defined in $L^{2}$. Another use of Strichartz's estimate gives us

$$
\left\|u-e^{i t \Delta} \psi_{+}\right\|_{L^{\infty}\left(\left([T, \infty), L^{2}\right)\right.} \leqslant C\|u\|_{L^{\rho}\left([T, \infty), L^{\gamma}\right)}^{p-1}\|u\|_{L^{q}\left(\left([T, \infty), L^{r}\right)\right.} \rightarrow 0
$$

as $T \rightarrow \infty$, which proves the scattering in $L^{2}$.

Now we assume that $u_{0} \in \mathcal{F} H^{1}$ and prove the first assertion. Take $t_{0} \in \mathbb{R}$ and interval $I \ni t_{0}$. Operating $J(t)$ to the integral form of (NLS) and applying (2.6), we obtain

$$
J(t) u(t)=e^{i\left(t-t_{0}\right) \Delta} J\left(t_{0}\right) u\left(t_{0}\right)-i \int_{t_{0}}^{t} e^{i(t-s) \Delta} J(s)\left(|u|^{p-1} u(s)\right) d s .
$$

Hence, it holds from Strichartz' estimate that

$$
\|J(t) u\|_{L^{q}\left(I, L^{r}\right)} \leqslant C\left\|J\left(t_{0}\right) u\left(t_{0}\right)\right\|_{L^{2}}+C\|u\|_{L^{\rho}\left(I, L^{\gamma}\right)}^{p-1}\|J(t) u\|_{L^{q}\left(I, L^{r}\right)} .
$$

Since $u \in L_{\text {loc }}^{2 / \delta(\gamma)}\left(\mathbb{R}, L^{\gamma}\right) \subset L_{\text {loc }}^{\rho}\left(\mathbb{R}, L^{\gamma}\right)$, it follows from (2.9) that the solution satisfies $J(t) u(t) \in L^{q}\left(I, L^{r}\right)$ at least on a small interval $I$ around $t=t_{0}$, provided $J\left(t_{0}\right) u\left(t_{0}\right) \in L^{2}$. Further, for the same interval $I$, we deduce from 
Strichartz' estimate that $J(t) u(t) \in C\left(I, L^{2}\right) \cap L_{\text {loc }}^{a}\left(I, L^{b}\right)$ for any admissible pair $(a, b)$. A similar argument shows that if the solution cannot extend beyond $t=T_{1}$ as a $\mathcal{F} H^{1}$-solution, that is, if $\|J(t) u(t)\|_{L^{q}\left((0, t), L^{r}\right)} \rightarrow \infty$ as $t \uparrow T_{1}$ holds for some time $T_{1}>0$ then $\|u\|_{L^{\rho}\left((0, t), L^{\gamma}\right)} \rightarrow \infty$ as $t \uparrow T_{1}$. However, such $T_{1}$ does not exist since $u \in L_{\text {loc }}^{\rho}\left(\mathbb{R}, L^{\gamma}\right)$ holds from the wellposedness in $L^{2}$. Thus, if $u_{0} \in \mathcal{F} H^{1}$ then $J(t) u(t) \in C\left(\mathbb{R}, L^{2}\right) \cap L_{\text {loc }}^{a}\left(\mathbb{R}, L^{b}\right)$ for any admissible pair $(a, b)$.

Let us proceed to the proof of the second assertion. Assume $u_{0} \in \mathcal{F} H^{1}$ and (2.7). Just as in the proof of $u(t) \in L^{a}\left((0, \infty), L^{b}\right)$, we see from (2.9) that $J(t) u(t) \in L^{a}\left((0, \infty), L^{b}\right)$ for any admissible pair $(a, b)$. Then, it holds that

$\left\|J(t) u(t)-e^{i t \Delta} x \psi\right\|_{L^{\infty}\left([T, \infty), L^{2}\right)} \leqslant C_{3}\|u\|_{L^{\rho}\left([T, \infty), L^{\gamma}\right)}^{p-1}\|J(t) u\|_{L^{q}\left([T, \infty), L^{r}\right)} \rightarrow 0$ as $T \rightarrow \infty$. This implies scattering in $\mathcal{F} H^{1}$ since

$$
\left\|J(t) u(t)-e^{i t \Delta} x \psi\right\|_{L^{2}}=\left\|x\left(e^{-i t \Delta} u(t)-\psi\right)\right\|_{L^{2}} .
$$

The case $u_{0} \in H^{1}$ is handled in a similar way.

Proposition 2.4. Suppose $p \in\left(p_{\mathrm{St}}, 1+\frac{4}{N}\right)$. Let $u_{0} \in \mathcal{F} H^{1}$ and let $u(t)$ be the corresponding global solution with $u(0)=u_{0}$. Then, $u$ scatters in $\mathcal{F} H^{1}$ for positive time if and only if (2.7) holds.

Proof. The "if part" is shown in Proposition 2.2. We shall prove the "only if part." Assume $u$ scatters in $\mathcal{F} H^{1}$. Since $u \in L_{\text {loc }}^{2 / \delta(\gamma)}\left(\mathbb{R}, L^{\gamma}\right) \subset L_{\text {loc }}^{\rho}\left(\mathbb{R}, L^{\gamma}\right)$, it suffices to show that $u \in L^{\rho}\left((1, \infty), L^{\gamma}\right)$. Since $e^{-i t \Delta} u(t)$ converges in $\mathcal{F} H^{1}$ as $t \rightarrow \infty$, we have

$$
\left\|e^{-i t \Delta} u(t)\right\|_{L^{\infty}\left((0, \infty), \mathcal{F} H^{1}\right)} \leqslant C .
$$

Hence, for $t>0$,

$$
\|u(t)\|_{L^{\gamma}} \leqslant C t^{-\delta(\gamma)}\left\|e^{-i t \Delta} u(t)\right\|_{\mathcal{F} H^{1}} \leqslant C t^{-\delta(\gamma)} .
$$

Notice that the right hand side belongs to $L_{t}^{\rho}((1, \infty))$ since $\rho \delta(\gamma)>1$ holds by assumption $p>p_{\mathrm{St}}$, which completes the proof.

Remark 2.5. By a similar manner, it is easy to see that $u(t)$ scatters in $\mathcal{F} H^{1}$ for negative time if and only if $\|u\|_{L^{\rho}\left((-\infty, 0), L^{\gamma}\right)}<\infty$.

\section{Proof of Theorem 1.7}

Although the existence of the minimal non-scattering solution (Theorem 1.3) has not been proven yet, we first establish Theorem 1.7 by showing that standing wave solutions do not satisfy the minimality property (property (2) of Theorem 1.3).

Lemma 3.1. Suppose $p \in\left(p_{\mathrm{St}}, 1+\frac{4}{N}\right)$. Let $u_{0} \in H^{1} \cap \mathcal{F} H^{1}$. If $E\left[u_{0}\right]<0$ then $u_{0} \in S_{+}^{c} \cap S_{-}^{c} \subset S^{c}$.

Proof. It is known that the energy is a conserved quantity. Hence, $E[u(t)]=$ $E\left[u_{0}\right]<0$ for all $t \in \mathbb{R}$. It then follows that $\|u(t)\|_{L^{p+1}}^{p+1} \geqslant-(p+1) E\left[u_{0}\right]>0$. This proves $\|u\|_{L^{\rho}\left((0, \infty), L^{\gamma}\right)}=\infty$ since $\gamma=p+1$ and $\rho<\infty$. Thus, by Proposition 2.4, $u_{0} \in S_{+}^{c}$. Similarly, $u_{0} \in S_{-}^{c}$. 
Theorem 3.2. Suppose $p \in\left(p_{\mathrm{St}}, 1+\frac{4}{N}\right)$. If $u_{0} \in H^{1} \cap \mathcal{F} H^{1}$ and if $E\left[u_{0}\right]<0$ then $\ell\left(u_{0}\right)>\inf \left\{\ell(f) \mid f \in \mathcal{F} H^{1} \backslash S\right\}$.

Proof. Suppose $u_{0} \in H^{1} \cap \mathcal{F} H^{1}$ and $E\left[u_{0}\right]<0$. Let $c>0$ be a real number. Since $E\left[c u_{0}\right]$ is continuous with respect to $c$ and since $E\left[u_{0}\right]<0$, there exists a number $c_{0} \in(0,1)$ such that $E\left[c_{0} u_{0}\right]<0$. Then, $c_{0} u_{0} \notin S$ by Lemma 3.1 . Moreover,

$$
\ell\left(u_{0}\right)>c_{0} \ell\left(u_{0}\right)=\ell\left(c_{0} u_{0}\right) \geqslant \inf \left\{\ell(f) \mid f \in \mathcal{F} H^{1} \backslash S\right\},
$$

which is the desired estimate.

By this theorem, if $u_{0}$ has negative energy then it cannot be equal to $u_{0, \mathrm{c}}$. The next well known lemma completes the proof of Theorem 1.7.

Lemma 3.3. If $\varphi \in H^{1}$ is a non-trivial solution to (1.3) then $\varphi \in H^{1} \cap \mathcal{F} H^{1}$ and $E[\varphi]<0$.

Proof. It is known that $\varphi$ decays exponentially. Hence, $\varphi \in \mathcal{F} H^{1}$. Further,

$$
E[\varphi]=\frac{N(p-1)-4}{2 N(p-1)}\|\nabla \varphi\|_{L^{2}}^{2}<0
$$

follows from Pohozaev's identity.

\section{LONG-Time PERTURBATION THEORY AND ITS APPLICATIONS}

In this section, we establish the following proposition.

Proposition 4.1 (long-time perturbation theory). Suppose $p_{\mathrm{St}}<p<1+\frac{4}{N}$. Let $\widetilde{u}(t, x)$ be a function defined on $[0, \infty) \times \mathbb{R}^{N}$ such that $e^{-i t \Delta} \widetilde{u} \in \mathcal{F} H^{1}$ for all $t \geqslant 0$. Define an error function e by

$$
e:=i \partial_{t} \widetilde{u}+\Delta \widetilde{u}+|\widetilde{u}|^{p-1} \widetilde{u} .
$$

Let $u_{0} \in \mathcal{F} H^{1}$ and let $u(t)$ be the corresponding unique global solution of (NLS) such that $u(0)=u_{0}$.

(1) For each $A>0$, there exists $\varepsilon_{0}=\varepsilon_{0}(A)>0$ such that the following holds; if

$$
\left\{\begin{array}{l}
\|\widetilde{u}\|_{L^{\rho}\left((0, \infty), L^{\gamma}\right)} \leqslant A \\
\|e\|_{L^{\widetilde{\rho}^{\prime}}\left((0, \infty), L^{\prime}\right)} \leqslant \varepsilon \\
\left\|e^{i t \Delta}(u(0)-\widetilde{u}(0))\right\|_{L^{\rho}\left((0, \infty), L^{\gamma}\right)} \leqslant \varepsilon
\end{array}\right.
$$

for $0<\varepsilon \leqslant \varepsilon_{0}$ then $u$ satisfies $\|u\|_{L^{\rho}\left((0, \infty), L^{\gamma}\right)} \leqslant A+\varepsilon^{\frac{1}{p}}$.

(2) There exist positive constants $C_{0}$ and $\delta$ such that if

$$
\begin{gathered}
R:=\left\|e^{i t \Delta}(u(0)-\widetilde{u}(0))\right\|_{L^{\rho}\left((0, \infty), L^{\gamma}\right)} \\
+\|\widetilde{u}\|_{L^{\rho}\left((0, \infty), L^{\gamma}\right)}+C_{0}\|e\|_{\left.L^{\tilde{\rho}^{\prime}\left((0, \infty), L^{\prime}\right.}\right)} \\
\text { satisfies } R< \\
\delta \text { then }\|u\|_{L^{\rho}\left((0, \infty), L^{\gamma}\right)} \leqslant 2 R .
\end{gathered}
$$

Let us now recall the following Gronwall-type inequality introduced in 8 , Lemma 8.1]. 
Lemma 4.2. Let $1 \leqslant \beta<\gamma \leqslant \infty, 0<T \leqslant \infty$, and let $f \in L^{\rho}((0, T))$, where $1 \leqslant \alpha<\infty$ is given by the relation $\alpha^{-1}=\beta^{-1}-\gamma^{-1}$. If $\eta \geqslant 0$ and $\varphi \in L_{\mathrm{loc}}^{\gamma}((0, T))$ satisfy

$$
\|\varphi\|_{L^{\gamma}((0, t))} \leqslant \eta+\|f \varphi\|_{L^{\beta}((0, t))}
$$

for all $0 \leqslant t \leqslant T$, then it holds that

$$
\|\varphi\|_{L^{\gamma}((0, t))} \leqslant \eta \Phi\left(\|f\|_{L^{\alpha}((0, t))}^{\alpha}\right)
$$

for all $0 \leqslant t \leqslant T$, where $\Phi(s):=2 \Gamma\left(2^{\alpha} s+3\right)$ and $\Gamma$ is the Gamma function.

Proof of Proposition 4.1. Let $w$ be defined by $u-\widetilde{u}=w$. Then $w$ solves the equation

$$
i \partial_{t} w+\Delta w+\left(|\widetilde{u}+w|^{p-1}(\widetilde{u}+w)-|\widetilde{u}|^{p-1} \widetilde{u}\right)-e=0 .
$$

By non-admissible Strichartz' estimate (2.4) and by (2.2), we have

$$
\begin{aligned}
& \text { 4.2) }\|w\|_{L^{\rho}\left((0, t), L^{\gamma}\right)} \leqslant\left\|e^{i t \Delta}\left(u_{0}-\widetilde{u}(0)\right)\right\|_{L^{\rho}\left((0, t), L^{\gamma}\right)} \\
& +C\|w\|_{L^{\rho}\left((0, t), L^{\gamma}\right)}^{p}+C\|\| \widetilde{u}(t)\left\|_{L^{\gamma}}^{p-1}\right\| w(t)\left\|_{L^{\gamma}}\right\|_{L^{\tilde{\rho}^{\prime}((0, t))}}+C\|e\|_{L^{\widetilde{\rho}^{\prime}\left((0, t), L^{\gamma^{\prime}}\right)}}
\end{aligned}
$$

for all $t \geqslant 0$.

Let us first prove the first assertion. Take $A>0$. Let $\varepsilon_{0}(A)>0$ be a number satisfying

$$
\frac{1}{\varepsilon_{0}^{p-1}} \geqslant 2\left\{(2 C+1) \Phi\left(C^{\frac{\rho}{p-1}} A^{\rho}\right)\right\}^{p},
$$

where $\Phi$ is a function given in Lemma 4.2. Assume (4.1). By assumption on $u_{0}$ and $e$,

$$
\begin{aligned}
& \|w\|_{L^{\rho}\left((0, t), L^{\gamma}\right)} \leqslant(C+1) \varepsilon+C\|w\|_{L^{\rho}\left((0, t), L^{\gamma}\right)}^{p} \\
& \quad+C\|\| \widetilde{u}(t)\left\|_{L^{\gamma}}^{p-1}\right\| w(t)\left\|_{L^{\gamma}}\right\|_{L^{\tilde{\rho}^{\prime}}((0, t))} .
\end{aligned}
$$

Take $T>0$ so that $\|w\|_{L^{\rho}\left((0, T), L^{\gamma}\right)}^{p} \leqslant \varepsilon \leqslant \varepsilon_{0}$. Then, we have

$$
\|\varphi\|_{L^{\rho}((0, t))} \leqslant(2 C+1) \varepsilon+\|f \varphi\|_{L^{\tilde{\rho}^{\prime}((0, t))}}
$$

for $0 \leqslant t \leqslant T$, where $\varphi(t)=\|w(t)\|_{L^{\gamma}}$ and $f(t)=C\|\widetilde{u}(t)\|_{L^{\gamma}}^{p-1}$. Apply Lemma 4.2 to yield

$$
\begin{aligned}
\|\varphi\|_{L^{\rho}((0, t))} & \leqslant(2 C+1) \varepsilon \Phi\left(\|f\|_{L^{\frac{\rho}{p-1}}((0, t))}^{\frac{\rho}{p-1}}\right) \\
& \leqslant(2 C+1) \varepsilon \Phi\left(C^{\frac{\rho}{p-1}}\|\widetilde{u}\|_{L^{\rho}((0, \infty))}^{\rho}\right) \leqslant \varepsilon(2 C+1) \Phi\left(C^{\frac{\rho}{p-1}} A^{\rho}\right) .
\end{aligned}
$$

By the assumption on $\varepsilon_{0}$,

$$
\|w\|_{L^{\rho}\left((0, T), L^{\gamma}\right)}^{p} \leqslant \varepsilon^{p}\left\{(2 C+1) \Phi\left(C^{\frac{\rho}{p-1}} A^{\rho}\right)\right\}^{p} \leqslant \frac{\varepsilon}{2}\left(\frac{\varepsilon}{\varepsilon_{0}}\right)^{p-1} \leqslant \frac{\varepsilon}{2} .
$$

By this estimate, we conclude that

$$
\sup _{T>0}\left\{\|w\|_{L^{\rho}\left((0, T), L^{\gamma}\right)}^{p} \leqslant \varepsilon\right\}=\infty .
$$

Hence, $\|w\|_{L^{\rho}\left((0, \infty), L^{\gamma}\right)} \leqslant \varepsilon^{1 / p}$. This completes the proof of the first assertion. 
We shall proceed to the proof of the second assertion. Now, let $\delta \in(0,1)$ to be chosen later. Let $C_{0}$ be the coefficient of the right hand side of (4.2). Assume $R<\delta$. Set $R_{0}:=R-\|\widetilde{u}\|_{L^{\rho}\left(I, L^{\gamma}\right)}$. Then, (4.2) gives us

$$
\|w\|_{L^{\rho}\left((0, t), L^{\gamma}\right)} \leqslant R_{0}+C\|w\|_{L^{\rho}\left((0, t), L^{\gamma}\right)}^{p}+C \delta^{p-1}\|w\|_{L^{\rho}\left((0, t), L^{\gamma}\right)}
$$

for all $t \geqslant 0$. Choosing $\delta$ so that $C \delta^{p-1} \leqslant 1 / 3$, we obtain

$$
\|w\|_{L^{\rho}\left((0, t), L^{\gamma}\right)} \leqslant \frac{3}{2} R_{0}+\frac{3}{2} C\|w\|_{L^{\rho}\left((0, t), L^{\gamma}\right)}^{p} .
$$

Now, set $f(x)=\frac{3}{2} R_{0}+\frac{3}{2} C x^{p}$. Remark that

$$
f\left(2 R_{0}\right)=\frac{3 R_{0}}{2}+\left(3 C\left(2 R_{0}\right)^{p-1}\right) R_{0} \leqslant R_{0}\left(\frac{3}{2}+3 C(2 \delta)^{p-1}\right) .
$$

Let $\delta$ be so small that $f\left(2 R_{0}\right)<2 R_{0}$, if necessary. For such $\delta$, (4.3) implies that $\|w\|_{L^{\rho}\left((0, t), L^{\gamma}\right)} \leqslant 2 R_{0}$ for every $t \geqslant 0$, showing $\|u\|_{L^{\rho}\left((0, \infty), L^{\gamma}\right)} \leqslant$ $\|\widetilde{u}\|_{L^{\rho}\left((0, \infty), L^{\gamma}\right)}+\|w\|_{L^{\rho}\left((0, \infty), L^{\gamma}\right)}<2 R$.

We introduce two consequent results. The first one is small data scattering.

Corollary 4.3 (small data scattering). Suppose $p_{\mathrm{St}}<p<1+\frac{4}{N}$. Let $u_{0} \in \mathcal{F} H^{1}$. There exists $\eta_{0}>0$ such that if $\left\|e^{i t \Delta} u_{0}\right\|_{L^{\rho}\left((0, \infty), L^{\gamma}\right)} \leqslant \eta_{0}$ then $u_{0} \in S_{+}$and the corresponding solution $u$ satisfies

$$
\|u\|_{L^{\rho}\left((0, \infty), L^{\gamma}\right)} \leqslant 2\left\|e^{i t \Delta} u_{0}\right\|_{L^{\rho}\left((0, \infty), L^{\gamma}\right)} .
$$

Further, there also exists $\eta_{1}>0$ such that if $\ell\left(u_{0}\right) \leqslant \eta_{1}$ then the same conclusion holds.

Proof. We apply the above proposition with $t_{0}=0$ and $\widetilde{u} \equiv 0$. Then, the former part follows immediately. The latter part is a consequence of Lemma 2.1 .

Remark 4.4. A similar result holds for negative time and for both time direction. We omit details.

We next show that the solution of (NLS) scatters for both time directions if the initial is sufficiently "oscillating." This result, which is an extension of [5], plays an important role in the proof of the profile decomposition lemma.

Proposition 4.5 (oscillating data scattering). Suppose $p_{\mathrm{St}}<p<1+\frac{4}{N}$. For any $\psi \in \mathcal{F} H^{1}$ and any $\varepsilon>0$, there exists $b_{0}>0$ such that if $|b|>b_{0}$ then

$$
\left\|e^{i t \Delta} e^{i b|x|^{2}} \psi\right\|_{L^{\rho}\left(\mathbb{R}, L^{\gamma}\right)} \leqslant \varepsilon .
$$

In particular, for any $\psi \in \mathcal{F} H^{1}$ there exists $b_{1}$ such that if $|b|>b_{1}$ then $e^{i b|x|^{2}} \psi \in S$.

Proof. The latter half is an immediate consequence of the former, by means of Corollary 4.3 (see also Remark 4.4). It is known that

$$
\left\|e^{i t \Delta} e^{i b|x|^{2}} \psi\right\|_{L^{\rho}\left((0, \infty), L^{\gamma}\right)} \rightarrow 0
$$


as $b \rightarrow \infty$ (see [5] and [4, Theorem 6.3.4]). By symmetry, this also implies $\left\|e^{i t \Delta} e^{i b|x|^{2}} \psi\right\|_{L^{\rho}\left((-\infty, 0), L^{\gamma}\right)} \rightarrow 0$ as $b \rightarrow-\infty$. Hence, it suffices to prove that

$$
\left\|e^{i t \Delta} e^{i b|x|^{2}} \psi\right\|_{L^{\rho}\left((0, \infty), L^{\gamma}\right)} \rightarrow 0
$$

as $b \rightarrow-\infty$. Let $b<0$. One verifies that

$$
e^{i t \Delta}\left(e^{i b|x|^{2}} \psi\right)(x)=e^{i \frac{b}{1+4 b t}|x|^{2}}(1+4 b t)^{-\frac{N}{2}}\left(e^{i \frac{t}{1+4 b t} \Delta} \psi\right)\left(\frac{x}{1+4 b t}\right) .
$$

Hence,

$$
\left\|e^{i t \Delta} e^{i b|x|^{2}} \psi\right\|_{L^{\gamma}}=|1+4 b t|^{-\delta(\gamma)}\left\|e^{i \frac{t}{1+4 b t} \Delta} \psi\right\|_{L^{\gamma}} .
$$

We estimate $L_{t}^{\rho}((0,1 / 4|b|))$ and $L_{t}^{\rho}((1 / 4|b|, \infty))$ individually. It holds that

$$
\begin{aligned}
\left\|e^{i t \Delta} e^{i b|x|^{2}} \psi\right\|_{L_{t}^{\rho}\left((0,1 / 4|b|), L^{\gamma}\right)}^{\rho} & =\int_{0}^{1 / 4|b|}|1+4 b t|^{-\rho \delta(\gamma)}\left\|e^{i \frac{t}{1+4 b t} \Delta} \psi\right\|_{L^{\gamma}}^{\rho} d t \\
& =\int_{0}^{\infty}(1-4 b s)^{\rho \delta(\gamma)-2}\left\|e^{i s \Delta} \psi\right\|_{L^{\gamma}}^{\rho} d s .
\end{aligned}
$$

Since $\rho \delta(\gamma)-2<0$, the integrand of the right hand side tends to zero as $b \rightarrow-\infty$ for each $s>0$. Further, Lemma 2.1 gives us

$$
\int_{0}^{\infty}(1-4 b s)^{\rho \delta(\gamma)-2}\left\|e^{i s \Delta} \psi\right\|_{L^{\gamma}}^{\rho} d s \leqslant\left\|e^{i s \Delta} \psi\right\|_{L_{s}^{\rho}\left((0, \infty), L^{\gamma}\right)}^{\rho} \leqslant C \ell(\psi)^{\rho}<\infty .
$$

We then see from Lebesgue's convergence theorem that

$$
\left\|e^{i t \Delta} e^{i b|x|^{2}} \psi\right\|_{L_{t}^{\rho}\left((0,1 / 4|b|), L^{\gamma}\right)} \rightarrow 0
$$

as $b \rightarrow-\infty$. Similarly, one deduces that

$$
\left\|e^{i t \Delta} e^{i b|x|^{2}} \psi\right\|_{L_{t}^{\rho}\left((1 / 4|b|, \infty), L^{\gamma}\right)}^{\rho}=\int_{-\infty}^{1 / 4 b}(1-4 b s)^{\rho \delta(\gamma)-2}\left\|e^{i s \Delta} \psi\right\|_{L^{\gamma}}^{\rho} d s .
$$

Fix a small number $a>0$. Then,

$\int_{-\infty}^{-a}(1-4 b s)^{\rho \delta(\gamma)-2}\left\|e^{i s \Delta} \psi\right\|_{L^{\gamma}}^{\rho} d s \leqslant(1-4 b a)^{\rho \delta(\gamma)-2}\left\|e^{i s \Delta} \psi\right\|_{L_{s}^{\rho}\left((-\infty, 0), L^{\gamma}\right)} \rightarrow 0$

as $b \rightarrow-\infty$. Let $\beta=\beta(a)>0$ to be chosen later. For sufficiently large $|b|$, we have $-a<\frac{1}{4 b}-\frac{\beta}{b}^{2}$. By Hölder's inequality,

$$
\begin{aligned}
& \int_{-a}^{\frac{1}{4 b}-\frac{\beta}{b^{2}}}(1-4 b s)^{\rho \delta(\gamma)-2}\left\|e^{i s \Delta} \psi\right\|_{L^{\gamma}}^{\rho} d s \\
& \leqslant\left(\int_{-a}^{\frac{1}{4 b}-\frac{\beta}{b^{2}}}(1-4 b s)^{-2} d s\right)^{\frac{1}{2}\left(1-\frac{\rho \delta(\gamma)}{2}\right)}\left\|e^{i s \Delta} \psi\right\|_{L^{2 / \delta(\gamma)}\left((-a, 0), L^{\gamma}\right)}^{\rho} \\
& \leqslant c_{1} \beta^{-\frac{1}{2}\left(1-\frac{\rho \delta(\gamma)}{2}\right)}\left\|e^{i s \Delta} \psi\right\|_{L^{2 / \delta(\gamma)}\left((-a, 0), L^{\gamma}\right)}^{\rho} .
\end{aligned}
$$


On the other hand, by Lemma 2.1

$$
\begin{aligned}
\int_{\frac{1}{4 b}-\frac{\beta}{b^{2}}}^{\frac{1}{4 b}}(1 & -4 b s)^{\rho \delta(\gamma)-2}\left\|e^{i s \Delta} \psi\right\|_{L^{\gamma}}^{\rho} d s \\
& \leqslant C \ell(\psi)^{\rho} \int_{\frac{1}{4 b}-\frac{\beta}{b^{2}}}^{\frac{1}{4 b}}(1-4 b s)^{\rho \delta(\gamma)-2}|s|^{-\rho \delta(\gamma)} d s \leqslant c_{2} \beta^{\rho \delta(\gamma)-1} \ell(\psi)^{\rho} .
\end{aligned}
$$

Combining these estimates, we obtain

$$
\begin{aligned}
& \int_{-a}^{\frac{1}{4 b}}(1-4 b s)^{\rho \delta(\gamma)-2}\left\|e^{i s \Delta} \psi\right\|_{L^{\gamma}}^{\rho} d s \\
& \leqslant c_{1}\left(\beta^{\frac{\delta(\gamma)}{2}-\frac{1}{\rho}}\left\|e^{i s \Delta} \psi\right\|_{L^{2 / \delta(\gamma)}\left((-a, 0), L^{\gamma}\right)}\right)^{\rho}+c_{2}\left(\beta^{\delta(\gamma)-\frac{1}{\rho}} \ell(\psi)\right)^{\rho} .
\end{aligned}
$$

Now, let $\beta=\left\|e^{i s \Delta} \psi\right\|_{L^{2 / \delta(\gamma)}\left((-a, 0), L^{\gamma}\right)}^{2 / \delta(\gamma)} \ell(\psi)^{-2 / \delta(\gamma)}$. Then,

$$
\int_{-a}^{\frac{1}{4 b}}(1-4 b s)^{\rho \delta(\gamma)-2}\left\|e^{i s \Delta} \psi\right\|_{L^{\gamma}}^{\rho} d s \leqslant C\left\|e^{i s \Delta} \psi\right\|_{L^{2 / \delta(\gamma)}\left((-a, 0), L^{\gamma}\right)}^{2 \rho-\frac{2}{\delta(\gamma)}} \ell(\psi)^{\frac{2}{\delta(\gamma)}-\rho} .
$$

Thus, we reach to the estimate

$$
\limsup _{b \rightarrow-\infty}\left\|e^{i t \Delta} e^{i b|x|^{2}} \psi\right\|_{L_{t}^{\rho}\left((0, \infty), L^{\gamma}\right)} \leqslant C\left\|e^{i s \Delta} \psi\right\|_{L^{2 / \delta(\gamma)}\left((-a, 0), L^{\gamma}\right)}^{2-\frac{2}{\rho \delta(\gamma)}} \ell(\psi)^{\frac{2}{\rho \delta(\gamma)}-1} .
$$

Recall that $a>0$ is arbitrary. Since $e^{i t \Delta} \psi \in L^{\frac{2}{\delta(\gamma)}}\left(\mathbb{R}, L^{\gamma}\right)$ by Strichartz' estimate and since $2 / \delta(\gamma)<\infty$ follows from $\gamma>2$, one sees that

$$
\left\|e^{i s \Delta} \psi\right\|_{L^{2 / \delta(\gamma)}\left((-a, 0), L^{\gamma}\right)} \rightarrow 0
$$

as $a \rightarrow 0$. Since $2-\frac{2}{\rho \delta(\gamma)}>0$ by (2.5), we finally obtain

$$
\limsup _{b \rightarrow-\infty}\left\|e^{i t \Delta} e^{i b|x|^{2}} \psi\right\|_{L_{t}^{\rho}\left((0, \infty), L^{\gamma}\right)}=0
$$

which completes the proof.

By this proposition, we obtain the following fact on the scattering set $S$.

Corollary 4.6. Suppose $p_{\mathrm{St}}<p<1+\frac{4}{N}$. The scattering set $S$ is an open subset of $\mathcal{F} H^{1}$ and unbounded in such a sense that

$$
\sup _{u_{0} \in S} \inf _{\mathbf{a} \in \mathbb{R}^{N}} \ell\left(u_{0}(\cdot-\mathbf{a})\right)=\sup _{u_{0} \in S} \inf _{\mathbf{a} \in \mathbb{R}^{N}}\left\|u_{0}(\cdot-\mathbf{a})\right\|_{\mathcal{F} H^{1}}=\infty .
$$

Proof. Openness immediately follows from Propositions 2.4 and 4.1. We prove unboundedness. Take a nontrivial radial function $\psi \in \mathcal{F} H^{1}$. Then, $\ell(\psi)=\inf _{\mathbf{a} \in \mathbb{R}^{N}} \ell\left(u_{0}(\cdot-\mathbf{a})\right)$. By Proposition 4.5, for any constant $C>0$ there exists $b_{0}=b_{0}(C) \in \mathbb{R}$ such that $e^{i b_{0}|x|^{2}}(C \psi) \in S$. Then, $\left\|e^{i b_{0}|x|^{2}} C \psi\right\|_{\mathcal{F} H^{1}} \geqslant$ $\ell\left(e^{i b_{0}|x|^{2}} C \psi\right)=C \ell(\psi)$. Since $C$ is arbitrary, unboundedness holds. 


\section{Profile decomposition}

This section is devoted to the proof of the profile decomposition lemma, which is one of the main tool for the proof of Theorem 1.3. A similar property for a sequence bounded in $\dot{H}^{1}$ is established in [16] (see also [2]), and applied in [15] to the study of NLS equation. In [7, 8], this is established for sequences bounded in $H^{1}$.

Proposition 5.1 (profile decomposition lemma). Suppose $p_{\mathrm{St}}<p<1+\frac{4}{N}$. Let $\left\{\phi_{n}\right\}_{n}$ be a bounded sequence in $\mathcal{F} H^{1}$. There exist a subsequence of $\left\{\phi_{n}\right\}$, which is denoted again by $\left\{\phi_{n}\right\}$, and sequences $\left\{\psi^{j}\right\}_{j} \subset \mathcal{F} H^{1},\left\{W_{n}^{j}\right\}_{n, j} \subset$ $\mathcal{F} H^{1}$, and $\left\{\xi_{n}^{j}\right\}_{n, j} \subset \mathbb{R}^{N}$ such that for every $l \geqslant 1$

$$
\phi_{n}=\sum_{j=1}^{l} e^{i \xi_{n}^{j} \cdot x} \psi^{j}+W_{n}^{l},
$$

in $\mathcal{F} H^{1}$ and,

$$
\left\|\phi_{n}\right\|_{\mathcal{F} \dot{H}^{s}}^{2}-\sum_{j=1}^{l}\left\|\psi^{j}\right\|_{\mathcal{F} \dot{H}^{s}}^{2}-\left\|W_{n}^{l}\right\|_{\mathcal{F} \dot{H}^{s}}^{2} \rightarrow 0
$$

as $n \rightarrow \infty$ for all $s \in[0,1]$. Furthermore, there exists $J \in[0, \infty]$ such that $\psi^{j} \equiv 0$ for all $j \geqslant 1$ if $J=0, \psi^{j} \not \equiv 0$ for all $j \geqslant 1$ if $J=\infty$, and

$$
\psi^{j} \not \equiv 0 \text { for } j \leqslant J, \quad \psi^{j} \equiv 0 \text { for } j>J
$$

holds if $J \in[1, \infty)$. For all $l \geqslant 1$ and $1 \leqslant i, j \leqslant J, i \neq j$,

$$
\lim _{n \rightarrow \infty}\left|\xi_{n}^{i}-\xi_{n}^{j}\right|=\infty
$$

In addition,

$$
\limsup _{n \rightarrow \infty}\left\|e^{i t \Delta} W_{n}^{l}\right\|_{L^{\rho}\left((0, \infty), L^{\gamma}\right)} \rightarrow 0
$$

as $l \rightarrow \infty$.

Let us begin the proof of Proposition 5.1 with the following lemma.

Lemma 5.2. Let $a>0$ and let $\left\{v_{n}\right\}_{n} \subset \mathcal{F} H^{1}$ satisfy

$$
\limsup _{n \rightarrow \infty}\left\|v_{n}\right\|_{\mathcal{F} H^{1}} \leqslant a<\infty \text {. }
$$

If

$$
\lim _{n \rightarrow \infty}\left\||t|^{\delta(\gamma)}\right\| e^{i t \Delta} v_{n}\left\|_{L^{\gamma}}\right\|_{L^{\infty}((0, \infty))}=A
$$

then there exist a subsequence, which denoted again by $\left\{v_{n}\right\}$, a function $\psi \in \mathcal{F} H^{1}$, and sequences $\left\{t_{n}\right\}_{n} \subset(0, \infty),\left\{\xi_{n}\right\}_{n} \subset \mathbb{R}^{N}$, and $\left\{W_{n}\right\}_{n} \subset \mathcal{F} H^{1}$ such that

$$
v_{n}=e^{-i \xi_{n} \cdot x} e^{-i t_{n}|x|^{2}} \psi+W_{n}
$$

with

$$
e^{i \xi_{n} \cdot x} e^{i t_{n}|x|^{2}} v_{n} \rightarrow \psi \quad \text { in } \mathcal{F} H^{1}
$$

or equivalently

$$
e^{i \xi_{n} \cdot x} e^{i t_{n}|x|^{2}} W_{n} \rightarrow 0 \quad \text { in } \mathcal{F} H^{1}
$$


as $n \rightarrow \infty$, and

$$
\left\|v_{n}\right\|_{\mathcal{F} \dot{H}^{s}}^{2}-\left\|W_{n}\right\|_{\mathcal{F} \dot{H}^{s}}^{2}-\|\psi\|_{\mathcal{F} \dot{H}^{s}}^{2} \rightarrow 0
$$

as $n \rightarrow \infty$ for all $s \in[0,1]$. Moreover, there exists a constant $c$ independent of $\left\{v_{n}\right\}_{n}, a$, and $A$ such that

$$
\|\psi\|_{\mathcal{F} H^{1}} \geqslant c a^{1-\frac{\gamma}{(\gamma-2)(1-\delta(\gamma))}} A^{\frac{\gamma}{(\gamma-2)(1-\delta(\gamma))}} .
$$

Proof. Let $\zeta$ be a smooth nonnegative radial function such that $\zeta(x)=1$ for $|x| \leqslant 1$ and $\zeta(x)=0$ for $|x| \geqslant 2$. Let

$$
\chi_{r}(t)=e^{i t \Delta} \zeta(x / r) e^{-i t \Delta} .
$$

One sees from Lemma 2.1 that

$$
\begin{aligned}
\left\|e^{i t \Delta} v_{n}-\chi_{r}(t) e^{i t \Delta} v_{n}\right\|_{L^{\gamma}} & =\left\|e^{i t \Delta}(1-\zeta(x / r)) v_{n}\right\|_{L^{\gamma}} \\
& \leqslant c_{0}|t|^{-\delta(\gamma)}\left\|(1-\zeta(x / r)) v_{n}\right\|_{L^{2}}^{1-\delta(\gamma)}\left\|x v_{n}\right\|_{L^{2}}^{\delta(\gamma)} \\
& \leqslant c_{0}|t|^{-\delta(\gamma)} r^{-(1-\delta(\gamma))}\left\|x v_{n}\right\|_{L^{2}} \\
& \leqslant c_{0}|t|^{-\delta(\gamma)} a r^{-(1-\delta(\gamma))} .
\end{aligned}
$$

We chose $r=\left(2 c_{0} a A^{-1}\right)^{1 /(1-\delta(\gamma))}$. This is possible because $\delta(\gamma)<1$. We then deduce from (5.7) that

$$
\begin{aligned}
& \sup _{t>0}|t|^{\delta(\gamma)}\left\|\chi_{r} e^{i t \Delta} v_{n}\right\|_{L^{\gamma}} \\
& \geqslant \sup _{t>0}\left(|t|^{\delta(\gamma)}\left\|e^{i t \Delta} v_{n}\right\|_{L^{\gamma}}-|t|^{\delta(\gamma)}\left\|e^{i t \Delta} v_{n}-\chi_{r} e^{i t \Delta} v_{n}\right\|_{L^{\gamma}}\right) \\
& \geqslant \sup _{t>0}\left(|t|^{\delta(\gamma)}\left\|e^{i t \Delta} v_{n}\right\|_{L^{\gamma}}-\frac{1}{2} A\right) \geqslant \frac{3}{4} A-\frac{1}{2} A=\frac{A}{4}
\end{aligned}
$$

for sufficiently large $n$. By Hölder's inequality, it holds that

$$
\begin{aligned}
|t|^{\delta(\gamma)}\left\|\chi_{r} e^{i t \Delta} v_{n}\right\|_{L^{\gamma}} & \leqslant|t|^{\delta(\gamma)}\left\|\chi_{r} e^{i t \Delta} v_{n}\right\|_{L^{2}}^{\frac{2}{\gamma}}\left\|\chi_{r} e^{i t \Delta} v_{n}\right\|_{L^{\infty}}^{1-\frac{2}{\gamma}} \\
& \leqslant a^{\frac{2}{\gamma}}\left(|t|^{\frac{N}{2}}\left\|\chi_{r} e^{i t \Delta} v_{n}\right\|_{L^{\infty}}\right)^{1-\frac{2}{\gamma}}
\end{aligned}
$$

for any $t \neq 0$. Combining above estimates, we obtain

$$
\sup _{t>0}|t|^{\frac{N}{2}}\left\|\chi_{r}(t) e^{i t \Delta} v_{n}\right\|_{L^{\infty}} \geqslant\left(\frac{A}{4 a^{2 / \gamma}}\right)^{\frac{\gamma}{\gamma-2}} \text {. }
$$

Hence, there exist sequences $\left\{t_{n}\right\}_{n} \subset(0, \infty)$ and $\left\{\xi_{n}\right\}_{n} \subset \mathbb{R}^{N}$ such that

$$
\left|4 t_{n}\right|^{-\frac{N}{2}}\left|\chi_{r}\left(\frac{1}{4 t_{n}}\right) e^{i \frac{1}{4 t_{n}} \Delta_{n}} v_{n}\right|\left(-\frac{\xi_{n}}{2 t_{n}}\right) \geqslant\left(\frac{A}{8 a^{2 / \gamma}}\right)^{\frac{\gamma}{\gamma-2}} .
$$

Set

$$
w_{n}(x):=e^{i \xi_{n} \cdot x} e^{i t_{n}|x|^{2}} v_{n}(x) .
$$

Since $\left\{w_{n}\right\}$ is bounded in $\mathcal{F} H^{1}$, we can extract a subsequence, denoted again by $\left\{w_{n}\right\}$, which converges weakly in $\mathcal{F} H^{1}$. Let the weak limit $\psi \in \mathcal{F} H^{1}$. 
Then, (5.9) holds. By the definition of $\chi_{r}$ and the integral representation of $e^{i \frac{1}{4 t_{n}} \Delta}$, one deduces that

$$
\begin{aligned}
& \left(4 t_{n}\right)^{-\frac{N}{2}}\left(\chi_{r}\left(\frac{1}{4 t_{n}}\right) e^{i \frac{1}{4 t_{n}} \Delta} v_{n}\right)\left(-\frac{\xi_{n}}{2 t_{n}}\right) \\
= & (4 \pi i)^{-\frac{N}{2}} \int_{\mathbb{R}^{N}} e^{i t_{n}\left|\xi_{n} / 2 t_{n}+y\right|^{2}} \zeta\left(\frac{y}{r}\right) v_{n}(y) \\
= & (4 \pi i)^{-\frac{N}{2}} e^{i \frac{\left|\xi_{n}\right|^{2}}{4 t_{n}}} \int_{\mathbb{R}^{N}} \zeta\left(\frac{y}{r}\right) w_{n}(y) d y .
\end{aligned}
$$

By extracting a subsequence, $e^{i \frac{\left|\xi_{n}\right|^{2}}{4 t_{n}}}$ converges. Denote the limit by $e^{i \theta}$. Then,

$$
\left(4 t_{n}\right)^{-\frac{N}{2}}\left(\chi_{r}\left(\frac{1}{4 t_{n}}\right) e^{i \frac{1}{t_{n}} \Delta} v_{n}\right)\left(-\frac{\xi_{n}}{2 t_{n}}\right) \rightarrow(4 \pi i)^{-\frac{N}{2}} e^{i \theta} \int_{\mathbb{R}^{N}} \zeta\left(\frac{y}{r}\right) \psi(y) d y .
$$

Therefore, for $n$ large enough,

$$
\left|4 t_{n}\right|^{-\frac{N}{2}}\left|\chi_{r}\left(\frac{1}{4 t_{n}}\right) e^{i \frac{1}{4 t_{n}} \Delta} v_{n}\right|\left(-\frac{\xi_{n}}{2 t_{n}}\right) \leqslant c_{1} r^{\frac{N}{2}}\|\psi\|_{L^{2}} .
$$

Thus, we conclude that

$$
\|\psi\|_{L^{2}} \geqslant C A^{\frac{\gamma}{\gamma-2}} a^{-\frac{2}{\gamma-2}} r^{-\frac{N}{2}}=c a^{1-\frac{\gamma}{(\gamma-2)(1-\delta(\gamma))}} A^{\frac{\gamma}{(\gamma-2)(1-\delta(\gamma))}},
$$

which yields (5.12) since $\|\psi\|_{L^{2}} \leqslant\|\psi\|_{\mathcal{F} H^{1}}$.

Set

$$
W_{n}:=v_{n}-e^{-i \xi_{n} \cdot x} e^{-i t_{n}|x|^{2}} \psi .
$$

Then, (5.10) immediately follows from (5.9). Further, for $s \in[0,1]$,

$$
\begin{aligned}
\left\|W_{n}\right\|_{\mathcal{F} \dot{H}^{s}}^{2} & =\left\|v_{n}\right\|_{\mathcal{F} \dot{H}^{s}}^{2}+\|\psi\|_{\mathcal{F} \dot{H}^{s}}^{2}-2 \operatorname{Re}\left\langle v_{n}, e^{-i \xi_{n} \cdot x} e^{-i t_{n}|x|^{2}} \psi\right\rangle_{\mathcal{F} \dot{H}^{s}} \\
& =\left\|v_{n}\right\|_{\mathcal{F} \dot{H}^{s}}^{2}+\|\psi\|_{\mathcal{F} \dot{H}^{s}}^{2}-2 \operatorname{Re}\left\langle w_{n}, \psi\right\rangle_{\mathcal{F} \dot{H}^{s}} .
\end{aligned}
$$

By means of (5.9), $\operatorname{Re}\left\langle w_{n}, \psi\right\rangle_{\mathcal{F} \dot{H}^{s}} \rightarrow\|\psi\|_{\mathcal{F} \dot{H}^{s}}^{2}$ as $n \rightarrow \infty$. This completes the proof of (5.11).

Lemma 5.3. Let $\left\{\tau_{n}\right\}_{n} \subset \mathbb{R}$ and $\left\{\xi_{n}\right\}_{n} \subset \mathbb{R}^{N}$ satisfy

$$
\left|\tau_{n}\right|+\left|\xi_{n}\right| \rightarrow \infty
$$

as $n \rightarrow \infty$. Then, if follows for all $\psi \in \mathcal{F} H^{1}$ that

$$
e^{i \tau_{n}|x|^{2}} e^{i x \cdot \xi_{n}} \psi \rightarrow 0 \quad \text { in } \mathcal{F} H^{1}
$$

as $n \rightarrow \infty$. Conversely, if $\left\{z_{n}\right\}_{N} \subset \mathcal{F} H^{1}$ satisfy

$$
z_{n} \rightarrow 0 \quad \text { in } \mathcal{F} H^{1}, \quad e^{i \tau_{n}|x|^{2}} e^{i x \cdot \xi_{n}} z_{n} \rightarrow \psi \quad \text { in } \mathcal{F} H^{1}
$$

as $n \rightarrow \infty$ for some $\left\{\tau_{n}\right\}_{n} \subset(0, \infty),\left\{\xi_{n}\right\}_{n} \subset \mathbb{R}^{N}$ and $\psi \in \mathcal{F} H^{1}, \psi \neq 0$, then (5.14) holds.

Proof. By the Fourier transform, it holds that, as $n \rightarrow \infty$,

$$
e^{i \tau_{n}|x|^{2}} e^{i x \cdot \xi_{n}} \psi \rightarrow 0 \quad \text { in } \mathcal{F} H^{1}
$$

is equivalent to

$$
e^{-i \tau_{n} \Delta}(\mathcal{F} \psi)\left(\cdot+\xi_{n}\right) \rightarrow 0 \quad \text { in } H^{1} .
$$

The lemma now follows from Lemma 5.3 of 8 . 
The next lemma is our first decomposition result, which is similar to those for $H^{1}$-bounded sequences obtained in [7, 8]. This decomposition involves quadratic oscillation.

Lemma 5.4. Under assumption of Proposition [5.1, there exist a subsequence of $\left\{\phi_{n}\right\}$, which is denoted again by $\left\{\phi_{n}\right\}$, and sequences $\left\{\psi^{j}\right\}_{j} \subset$ $\mathcal{F} H^{1},\left\{W_{n}^{j}\right\}_{n, j} \subset \mathcal{F} H^{1},\left\{t_{n}^{j}\right\}_{n, j} \subset(-\infty, 0),\left\{\bar{t}^{j}\right\}_{j} \subset[-\infty, 0]$, and $\left\{\xi_{n}^{j}\right\}_{n, j} \subset$ $\mathbb{R}^{N}$ such that for every $l \geqslant 1$

$$
\phi_{n}=\sum_{j=1}^{l} e^{i \xi_{n}^{j} \cdot x} e^{i t_{n}^{j}|x|^{2}} \psi^{j}+W_{n}^{l},
$$

in $\mathcal{F} H^{1}$ and, as $n \rightarrow \infty$,

$$
\begin{gathered}
t_{n}^{j} \rightarrow \bar{t}^{j}, \\
\left\|\phi_{n}\right\|_{\mathcal{F} \dot{H}^{s}}^{2}-\sum_{j=1}^{l}\left\|\psi^{j}\right\|_{\mathcal{F} \dot{H}^{s}}^{2}-\left\|W_{n}^{j}\right\|_{\mathcal{F} \dot{H}^{s}}^{2} \rightarrow 0
\end{gathered}
$$

for all $s \in[0,1]$. Furthermore, there exists $J \in \mathbb{N} \cup\{\infty\}$ such that $\psi^{j} \equiv 0$ for all $j \geqslant 1$ if $J=0, \psi^{j} \not \equiv 0$ for all $j \geqslant 1$ if $J=\infty$, and

$$
\psi^{j} \not \equiv 0 \text { for } j \leqslant J, \quad \psi^{j} \equiv 0 \text { for } j>J
$$

if $J \in[1, \infty)$. For all $l \geqslant 1$ and $1 \leqslant i, j \leqslant J, i \neq j$,

$$
\lim _{n \rightarrow \infty}\left|t_{n}^{i}-t_{n}^{i}\right|+\left|x_{n}^{i}-x_{n}^{j}\right|=\infty \text {. }
$$

In addition,

$$
\limsup _{n \rightarrow \infty}\left\|e^{i t \Delta} W_{n}^{l}\right\|_{L^{\rho}\left((0, \infty), L^{\gamma}\right)} \rightarrow 0
$$

as $l \rightarrow \infty$.

Remark 5.5. As shown in [7, 8], a $H^{1}$-bounded sequence $\left\{\varphi_{n}\right\}_{n}$ is decomposed, up to a subsequence, into a form

$$
\varphi_{n}=\sum_{j=1}^{l} e^{-i t_{n}^{j} \Delta} \psi^{j}\left(x-x_{n}^{j}\right)+W_{n}^{j} .
$$

Our decomposition (5.16) is similar to the Fourier transform of this in such a sense that the both give profiles of the same form. However, it is not cleat whether the estimate (5.21) follows from this correspondence.

Proof. Let

$$
a=\limsup _{n \rightarrow \infty}\left\|\phi_{n}\right\|_{\mathcal{F} H^{1}}>0
$$

Let $W_{n}^{0}=\phi_{n}$. We construct by induction on $l$ the various sequences so that for every $1 \leqslant j \leqslant l$,

$$
W_{n}^{j-1}=e^{i \xi_{n}^{j} \cdot x} e^{i t_{n}^{j}|x|^{2}} \psi^{j}+W_{n}^{j}
$$

for $n \geqslant 1$ and

$$
\begin{gathered}
t_{n}^{j} \rightarrow \bar{t}^{j} \in[-\infty, 0], \\
e^{-i \xi_{n}^{j} \cdot x} e^{-i t_{n}^{j}|x|^{2}} W_{n}^{j-1} \rightarrow \psi^{j}, \quad e^{-i \xi_{n}^{j} \cdot x} e^{-i t_{n}^{j}|x|^{2}} W_{n}^{j} \rightarrow 0
\end{gathered}
$$


in $\mathcal{F} H^{1}$ as $n \rightarrow \infty$,

$$
\left\|W_{n}^{j-1}\right\|_{\mathcal{F} \dot{H}^{s}}^{2}-\left\|\psi^{j}\right\|_{\mathcal{F} \dot{H}^{s}}^{2}-\left\|W_{n}^{j}\right\|_{\mathcal{F} \dot{H}^{s}}^{2} \rightarrow 0
$$

as $n \rightarrow \infty$ for all $s \in[0,1]$, and

$$
\begin{gathered}
\left\||t|^{\delta(\gamma)}\right\| e^{i t \Delta} W_{n}^{j-1}\left\|_{L^{\gamma}}\right\|_{L^{\infty}((0, \infty))} \rightarrow A_{j}, \\
\left\|\psi^{j}\right\|_{\mathcal{F} H^{1}} \geqslant \nu(a) A_{j}^{\frac{\gamma}{(\gamma-2)(1-\delta(\gamma))}} .
\end{gathered}
$$

For $l=1$, we set

$$
A_{1}=\limsup _{n \rightarrow \infty}\left\||t|^{\delta(\gamma)}\right\| e^{i t \Delta} \phi_{n}\left\|_{L^{\gamma}}\right\|_{L^{\infty}((0, \infty))} .
$$

We can extract a subsequence so that

$$
\lim _{n \rightarrow \infty}\left\||t|^{\delta(\gamma)}\right\| e^{i t \Delta} \phi_{n}\left\|_{L^{\gamma}}\right\|_{L^{\infty}((0, \infty))}=A_{1} .
$$

We apply Lemma 5.2 with $v_{n}=W_{n}^{0}:=\phi_{n}$. Then, we obtain $\psi^{1},\left\{t_{n}^{1}\right\}_{n}$, $\left\{\xi_{n}\right\}_{n}$, and $\left\{W_{n}^{1}\right\} \subset \mathcal{F} H^{1}$ which ensure (5.22), (5.24), (5.25), (5.26), and (5.27). Moreover, by extracting a subsequence if necessary, we claim that $t_{n}^{1} \rightarrow \bar{t}^{1} \in[-\infty, 0]$. This is (5.23).

Fix $l \geqslant 2$ and suppose that $\left\{t_{n}^{j}\right\}_{n}, \bar{t}^{j},\left\{\xi_{n}^{j}\right\}_{n}, \psi^{j}$, and $\left\{W_{n}^{j}\right\}_{n}$ are successfully constructed for all $j \leqslant l-1$. Set

$$
A_{l}=\limsup _{n \rightarrow \infty}\left\||t|^{\delta(\gamma)}\right\| e^{i t \Delta} W_{n}^{l-1}\left\|_{L^{\gamma}}\right\|_{L^{\infty}((0, \infty))} .
$$

By extracting a subsequence, we can replace limsup by lim, which implies (5.26) holds. We now apply Lemma 5.2 with $v_{n}=W_{n}^{l-1}$. Then, as in the case $l=1$, we obtain $\left\{t_{n}^{l}\right\}_{n}, \bar{t}^{l},\left\{\xi_{n}^{l}\right\}, \psi^{l}$, and $\left\{W_{n}^{l}\right\}_{n}$ so that (5.22), (5.23), (5.24), and (5.25) hold with $j=l$. Summing up the equation (5.25) with respect to $j$ yields

$$
\sum_{j=1}^{l}\left\|\psi^{j}\right\|_{\mathcal{F} H^{1}}^{2} \leqslant a^{2}
$$

showing in particular $\left\|\psi^{l}\right\|_{\mathcal{F} H^{1}} \leqslant a$. By (5.12), we have (5.27) for $j=l$.

We shall check the sequences we have constructed satisfy the desired properties. The properties (5.16), (5.17), and (5.18) follow immediately from (5.22), (5.23), (5.25), and $W_{n}^{0}=\phi_{n}$. The estimates (5.25) and (5.27) infer that

$$
\sum_{j=1}^{\infty} A_{j}^{\frac{2 \gamma}{(\gamma-2)(1-\delta(\gamma))}} \leqslant C(a) \sum_{j=1}^{\infty}\left\|\psi^{j}\right\|_{\mathcal{F} H^{1}}^{2}<\infty,
$$

showing that $A_{l} \rightarrow 0$ as $l \rightarrow \infty$. Now, it holds for any $T>0$ that

$$
\begin{aligned}
\left\|e^{i t \Delta} W_{n}^{l}\right\|_{L^{\rho}\left((0, \infty), L^{\gamma}\right)} \leqslant & T^{\frac{1}{\rho}-\frac{1}{\eta}}\left\|e^{i t \Delta} W_{n}^{l}\right\|_{L^{\eta}\left((0, T), L^{\gamma}\right)} \\
& +T^{\frac{1}{\rho}-\delta(\gamma)}\left\||t|^{\delta(\gamma)}\right\| e^{i t \Delta} W_{n}^{l}\left\|_{L^{\gamma}}\right\|_{L^{\infty}((T, \infty))},
\end{aligned}
$$


where $\eta=2 / \delta(\gamma)$. Minimizing the right hand side with respect to $T$ and applying Strichartz' estimate, we obtain

$$
\left\|e^{i t \Delta} W_{n}^{l}\right\|_{L^{\rho}\left((0, \infty), L^{\gamma}\right)} \leqslant C\left\|W_{n}^{l}\right\|_{L^{2}}^{2-\frac{2}{\rho(\gamma)}}\left\||t|^{\delta(\gamma)}\right\| e^{i t \Delta} W_{n}^{l}\left\|_{L^{\gamma}}\right\|_{L^{\infty}((0, \infty))}^{\frac{2}{\rho \delta(\gamma)}-1} .
$$

Thus, $A_{l} \rightarrow 0$ as $l \rightarrow \infty$ implies

$$
\lim _{n \rightarrow \infty}\left\|e^{i t \Delta} W_{n}^{l}\right\|_{L^{\rho}\left((0, \infty), L^{\gamma}\right)} \rightarrow 0
$$

as $l \rightarrow \infty$, which is (5.21). Let us next show the existence of $J \in[0, \infty]$. If $\psi^{j_{0}}=0$ for some $j_{0} \geqslant 1$ in the above procedure, then $A_{j_{0}}=0$ holds by (5.27). All the properties (except for (5.20)) are then satisfied with the choice $\psi^{l}=0$ and $W_{n}^{l}=W_{n}^{j_{0}}$ for all $l \geqslant j_{0}$. Hence, we let $J=j_{0}-1$ in this case. If such $j_{0}$ does not exist then we let $J=\infty$. Let us proceed to the proof of (5.20). Assume $J \geqslant 2$ and take $2 \leqslant l \leqslant J$. We shall show by induction on $k$ that

$$
\left|t_{n}^{l}-t_{n}^{l-k}\right|+\left|\xi_{n}^{l}-\xi_{n}^{l-k}\right| \rightarrow \infty
$$

as $n \rightarrow \infty$ for all $1 \leqslant k \leqslant l-1$. First consider the case $k=1$. By (5.24) with $j=l-1, l$, it follows that

$$
e^{-i \xi_{n}^{l-1} \cdot x} e^{-i t_{n}^{l-1}|x|^{2}} W_{n}^{l-1} \rightarrow 0, \quad e^{-i \xi_{n}^{l} \cdot x} e^{-i t_{n}^{l}|x|^{2}} W_{n}^{l-1} \rightarrow \psi^{l}
$$

in $\mathcal{F} H^{1}$ as $n \rightarrow \infty$. Apply Lemma 5.3 with $z_{n}=e^{-i \xi_{n}^{l-1} \cdot x} e^{-i t_{n}^{l-1}|x|^{2}} W_{n}^{l-1}$ to yield

$$
\left|t_{n}^{l}-t_{n}^{l-1}\right|+\left|\xi_{n}^{l}-\xi_{n}^{l-1}\right| \rightarrow \infty
$$

as $n \rightarrow \infty$. The proof is done when $J=2$. Hence we let $J \geqslant 3$ and $l \geqslant 3$. Suppose that (5.28) is true for all $1 \leqslant k \leqslant k_{0}-1$, where $k_{0} \in[2, l-1]$. Then, by (5.22),

$$
W_{n}^{l-1}-W_{n}^{l-k_{0}}=\sum_{j=l-k_{0}+1}^{l-1} e^{i \xi_{n}^{j} \cdot x} e^{i t_{n}^{j}|x|^{2}} \psi^{j} .
$$

This implies

$$
\begin{aligned}
e^{-i \xi_{n}^{l} \cdot x} e^{-i t_{n}^{l}|x|^{2}} & W_{n}^{l-k_{0}} \\
& =e^{-i \xi_{n}^{l} \cdot x} e^{-i t_{n}^{l}|x|^{2}} W_{n}^{l-1}-\sum_{j=l-k_{0}+1}^{l-1} e^{i\left(\xi_{n}^{j}-\xi_{n}^{l}\right) \cdot x} e^{i\left(t_{n}^{j}-t_{n}^{l}\right)|x|^{2}} \psi^{j} .
\end{aligned}
$$

One then sees from (5.24) with $j=l$ that $e^{-i \xi_{n}^{l} \cdot x} e^{-i t_{n}^{l}|x|^{2}} W_{n}^{l-1} \rightarrow \psi^{l}$ in $\mathcal{F} H^{1}$ as $n \rightarrow \infty$. Moreover, it follows from the former part of Lemma 5.3 and assumption of induction that

$$
\sum_{j=l-k_{0}+1}^{l-1} e^{i\left(\xi_{n}^{j}-\xi_{n}^{l}\right) \cdot x} e^{i\left(t_{n}^{j}-t_{n}^{l}\right)|x|^{2}} \psi^{j} \rightarrow 0
$$

in $\mathcal{F} H^{1}$ as $n \rightarrow \infty$. Hence, as $n \rightarrow \infty$,

$$
e^{-i \xi_{n}^{l} \cdot x} e^{-i t_{n}^{l}|x|^{2}} W_{n}^{l-k_{0}} \rightarrow \psi^{l} .
$$


On the other hand, by (5.24) with $j=l-k_{0}$, we have

$$
e^{-i \xi_{n}^{l-k_{0}} \cdot x} e^{-i t_{n}^{l-k_{0}}|x|^{2}} W_{n}^{l-k_{0}} \rightarrow 0
$$

in $\mathcal{F} H^{1}$ as $n \rightarrow \infty$. The latter part of Lemma 5.3 shows $\left|t_{n}^{l}-t_{n}^{l-k_{0}}\right|+\mid \xi_{n}^{l}-$ $\xi_{n}^{l-k_{0}} \mid \rightarrow \infty$ as $n \rightarrow \infty$, which is (5.28) with $l=k_{0}$. Hence, (5.28) is true for all $1 \leqslant k \leqslant l-1$ and $2 \leqslant l \leqslant J$, which completes the proof of (5.20).

We finally prove Proposition 5.1. To do so, it is essential to show that we can let $\bar{t}^{j}>-\infty$ for all $j$ in Lemma 5.4. Heart of matter is that if $t_{n}^{j} \rightarrow-\infty$ as $n \rightarrow \infty$ then $e^{i t_{n}^{j}|x|^{2}} \psi^{j}$ becomes a remainder term by means of Proposition 4.5.

Proof of Proposition 5.1. We first apply Lemma 5.4 to obtain

$$
\phi_{n}=\sum_{j=1}^{l} e^{i \xi_{n}^{j} \cdot x} e^{i t_{n}^{j}|x|^{2}} \varphi^{j}+w_{n}^{l}
$$

Set $L=\left\{l \in[1, J] \mid \bar{t}^{l}>-\infty\right\}$. If $L=\emptyset$ then the result follows with $\psi^{j}=0$

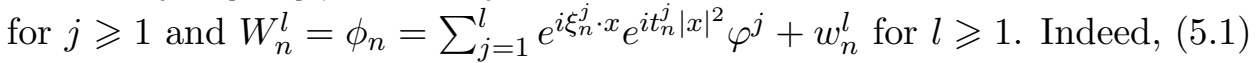
and (5.2) are obvious, $J=0$, and we see from Proposition 4.5 that

$$
\limsup _{n \rightarrow \infty}\left\|e^{i t \Delta} W_{n}^{l}\right\|_{L^{\rho}\left((0, \infty), L^{\gamma}\right)}=\limsup _{n \rightarrow \infty}\left\|e^{i t \Delta} w_{n}^{l}\right\|_{L^{\rho}\left((0, \infty), L^{\gamma}\right)} \rightarrow 0
$$

as $l \rightarrow \infty$, which is (5.5).

Consider the case $L \neq \emptyset$. We may first assume $L$ is not a finite set. We number the elements of $L$ as $L=\left\{j_{1}, j_{2}, j_{3}, \ldots\right\}$ in such a way that $j_{k}<j_{l}$ as long as $k<l$. For each $l \geqslant 1$, we define

$$
\psi^{l}=e^{i \bar{t}^{j_{l}}|x|^{2}} \varphi^{j_{l}}
$$

and

$$
W_{n}^{l}=\sum_{k=1}^{l} e^{i \xi_{n}^{j_{k}} \cdot x}\left(e^{i t_{n}^{j_{k}}|x|^{2}} \varphi^{j_{k}}-\psi^{k}\right)+\sum_{j \leqslant j_{l}, j \notin L} e^{i \xi_{n}^{j} \cdot x} e^{i t_{n}^{j}|x|^{2}} \varphi^{j}+w_{n}^{j_{l}} .
$$

Then, (5.1) holds. Remark that $e^{i \xi_{n}^{j_{l}} \cdot x}\left(e^{i t_{n}^{j_{l}}|x|^{2}} \varphi^{j}-\psi^{l}\right) \rightarrow 0$ strongly in $\mathcal{F} H^{1}$ as $n \rightarrow \infty$. Therefore, by Lemma 2.1, we have

$$
\limsup _{n \rightarrow \infty}\left\|e^{i t \Delta} W_{n}^{l}\right\|_{L^{\rho}\left((0, \infty), L^{\gamma}\right)}=\limsup _{n \rightarrow \infty}\left\|e^{i t \Delta} w_{n}^{j_{l}}\right\|_{L^{\rho}\left((0, \infty), L^{\gamma}\right)} \rightarrow 0
$$

as $l \rightarrow \infty$. Thus, (5.5) holds. Further, as in the proof of Lemma 5.4, one sees that

$$
\left\|W_{n}^{l}\right\|_{\mathcal{F} \dot{H}^{s}}^{2}-\sum_{j \leqslant j_{l}, j \notin L}\left\|\varphi^{j}\right\|_{\mathcal{F} \dot{H}^{s}}^{2}-\left\|w_{n}^{j_{l}}\right\|_{\mathcal{F} \dot{H}^{s}}^{2} \rightarrow 0
$$


as $n \rightarrow \infty$ for any $s \in[0,1]$. Therefore,

$$
\begin{aligned}
& \left\|\phi_{n}\right\|_{\mathcal{F} \dot{H}^{s}}^{2}-\sum_{j=1}^{l}\left\|\psi^{j}\right\|_{\mathcal{F} \dot{H}^{s}}^{2}-\left\|W_{n}^{l}\right\|_{\mathcal{F} \dot{H}^{s}}^{2} \\
= & \left(\left\|\phi_{n}\right\|_{\mathcal{F}^{\dot{H}^{s}}}^{2}-\sum_{j=1}^{j_{l}}\left\|\varphi^{j}\right\|_{\mathcal{F} \dot{H}^{s}}^{2}-\left\|w_{n}^{j_{l}}\right\|_{\mathcal{F} \dot{H}^{s}}^{2}\right) \\
& -\left(\left\|W_{n}^{l}\right\|_{\mathcal{F} \dot{H}^{s}}^{2}-\sum_{j \leqslant j_{l}, j \notin L}\left\|\varphi^{j}\right\|_{\mathcal{F} \dot{H}^{s}}^{2}-\left\|w_{n}^{j_{l}}\right\|_{\mathcal{F} \dot{H}^{s}}^{2}\right) \rightarrow 0
\end{aligned}
$$

as $l \rightarrow \infty$, showing (5.2). The property (5.4) is an immediate consequence of (5.20) and the fact that $\bar{t}_{j}>-\infty$ for $j \in L$.

If $L$ is a finite set then it suffices to define $\psi^{l}$ and $W_{n}^{l}$ for $l>\sharp L$ as follows; $\psi^{l}=0$ and $W_{n}^{l}=w_{n}^{\max L}$. Then, (5.3) is trivial. Remark that

$$
\limsup _{n \rightarrow \infty}\left\|e^{i t \Delta} w_{n}^{\max L}\right\|_{L^{\rho}\left((0, \infty), L^{\gamma}\right)}=\limsup _{n \rightarrow \infty}\left\|e^{i t \Delta} w_{n}^{l}\right\|_{L^{\rho}\left((0, \infty), L^{\gamma}\right)} \rightarrow 0
$$

as $l \rightarrow \infty$ by Proposition 4.5, that is, $\lim _{4 \sup _{n \rightarrow \infty}}\left\|e^{i t \Delta} w_{n}^{\max L}\right\|_{L^{\rho}\left((0, \infty), L^{\gamma}\right)}=$ 0. Hence (5.5) holds.

\section{Proof of Theorem 1.3}

Let us define

$$
\ell_{c}:=\inf \left\{\ell(f) \mid f \in \mathcal{F} H^{1} \backslash S_{+}\right\} .
$$

By Corollary 4.3, there exists a constant $\eta_{1}>0$ such that $\ell_{c} \geqslant \eta_{1}$. On the other hand, since $Q \in \mathcal{F} H^{1} \backslash S_{+}$, we have $\ell_{c} \leqslant \ell(Q)<\infty$, where $Q$ is a ground state. Thus, $\ell_{c} \in(0, \infty)$.

The main step of the proof is the following.

Lemma 6.1. Let $\left\{u_{0, n}\right\}_{n} \subset \mathcal{F} H^{1}$ be a sequence satisfying $u_{0, n} \notin S_{+}$and $\ell\left(u_{0, n}\right) \leqslant \ell_{c}+\frac{1}{n}$. Assume $\left\|u_{0, n}\right\|_{L^{2}}=1$. Then, there exist a subsequence of $\left\{u_{0, n}\right\}_{n}$, which is denoted again by $\left\{u_{0, n}\right\}_{n}$, a function $\psi \in \mathcal{F} H^{1}$ with $\|\psi\|_{L^{2}}=1$ and $\ell(\psi)=\ell_{c}$, and sequences $\left\{W_{n}\right\}_{n} \subset \mathcal{F} H^{1}$ and $\left\{\xi_{n}\right\}_{n} \subset \mathbb{R}^{N}$ such that

$$
u_{0, n}=e^{i \xi_{n} \cdot x} \psi+W_{n}
$$

and

$$
\left\|W_{n}\right\|_{\mathcal{F} H^{1}} \rightarrow 0
$$

as $n \rightarrow \infty$.

Proof. It holds by assumption $\left\|u_{0, n}\right\|_{L^{2}}=1$ that

$$
\limsup _{n \rightarrow \infty}\left\|x u_{0, n}\right\|_{L^{2}}^{\frac{2}{p-1}-\frac{N}{2}} \leqslant \ell_{c} .
$$

Hence, $u_{0, n}$ is uniformly bounded in $\mathcal{F} H^{1}$. By profile decomposition (Proposition 5.11), there exist a subsequence of $\left\{u_{0, n}\right\}$, which is denoted again by 
$\left\{u_{0, n}\right\}$, and sequences $\left\{\psi^{j}\right\}_{j} \subset \mathcal{F} H^{1},\left\{W_{n}^{j}\right\}_{n, j} \subset \mathcal{F} H^{1}$, and $\left\{\xi_{n}^{j}\right\}_{n, j} \subset \mathbb{R}^{N}$ such that for every $l \geqslant 1$

$$
u_{0, n}=\sum_{j=1}^{l} e^{i \xi_{n}^{j} \cdot x} \psi^{j}+W_{n}^{l},
$$

in $\mathcal{F} H^{1}$ and, as $n \rightarrow \infty$,

$$
\left\|u_{0, n}\right\|_{\mathcal{F} \dot{H}^{s}}^{2}-\sum_{j=1}^{l}\left\|\psi^{j}\right\|_{\mathcal{F} \dot{H}^{s}}^{2}-\left\|W_{n}^{j}\right\|_{\mathcal{F} \dot{H}^{s}}^{2} \rightarrow 0
$$

for all $s \in[0,1]$. Furthermore, there also exists $J \in \mathbb{N} \cup\{\infty\}$ such that (5.3) holds. For all $l \geqslant 1$ and $1 \leqslant i, j<J, i \neq j$,

$$
\lim _{n \rightarrow \infty}\left|\xi_{n}^{i}-\xi_{n}^{j}\right|=\infty
$$

In addition,

$$
\limsup _{n \rightarrow \infty}\left\|e^{i t \Delta} W_{n}^{l}\right\|_{L^{\rho}\left((0, \infty), L^{\gamma}\right)} \rightarrow 0
$$

as $l \rightarrow \infty$. Property (6.5) with $s=0$ yields

$$
1-\sum_{j=1}^{l}\left\|\psi^{j}\right\|_{L^{2}}^{2}-\left\|W_{n}^{j}\right\|_{L^{2}}^{2} \rightarrow 0
$$

In particular, $\sum_{j=1}^{l}\left\|\psi^{j}\right\|_{L^{2}}^{2} \leqslant 1$. Since $l$ is arbitrary, we have

$$
\sum_{j=1}^{\infty}\left\|\psi^{j}\right\|_{L^{2}}^{2} \leqslant 1
$$

Repeating this argument with $s=1$, we deduce from (6.3) that

$$
\begin{aligned}
\sum_{j=1}^{\infty}\left\|x \psi^{j}\right\|_{L^{2}}^{2} & \leqslant \limsup _{n \rightarrow \infty}\left\|x u_{0, n}\right\|_{L^{2}}^{2} \\
& \leqslant \ell_{c}^{2\left(\frac{2}{p-1}-\frac{N}{2}\right)^{-1}} .
\end{aligned}
$$

Thus, $\ell\left(\psi^{j}\right) \leqslant \ell_{c}$.

We now claim that there exists $j_{0}$ such that $\ell\left(\psi^{j_{0}}\right)=\ell_{c}$. This claim completes the proof. Indeed, if such $j_{0}$ exists then the inequality

$$
\left\|x \psi^{j_{0}}\right\|_{L^{2}} \leqslant \ell_{c}^{\left(\frac{2}{p-1}-\frac{N}{2}\right)^{-1}}
$$

which follows from (6.9), yields $\left\|\psi^{j_{0}}\right\|_{L^{2}} \geqslant 1$. On the other hand, (6.8) gives $\left\|\psi^{j_{0}}\right\|_{L^{2}} \leqslant 1$. Therefore, $\left\|\psi^{j_{0}}\right\|_{L^{2}}=1$. Further, it then follows from (6.8) that $\psi^{j} \equiv 0$ for all $j \neq j_{0}$. We hence obtain (6.1) with $\psi=\psi^{j_{0}}$ and $W_{n}=W_{n}^{j_{0}}$. The property (6.2) immediately follows from (6.5) with $s=0,1$. Thus, the lemma is proven.

Let us show the claim. We assume for contradiction that $\ell\left(\psi^{j}\right)<\ell_{c}$ for all $j$. Then, $\psi^{j} \in S_{+}$by definition of $\ell_{c}$. Let $V_{j}$ be a solution of (NLS) with 
$\left.V_{j}\right|_{t=0}=\psi^{j}$ and let

$$
\widetilde{u}_{n}^{l}(t, x):=\sum_{j=1}^{l} V_{j}\left(t, x-t \xi_{n}^{j}\right) e^{i \xi_{n}^{j} \cdot x} e^{i \frac{t}{2}\left|\xi_{n}^{j}\right|^{2}} .
$$

It follows that

$$
\begin{aligned}
\left(i \partial_{t}+\Delta\right) \widetilde{u}_{n}^{l}(t) & =\sum_{j=1}^{l}\left(i \partial_{t}+\Delta\right)\left(V_{j}\left(t, x-t \xi_{n}^{j}\right) e^{i \xi_{n}^{j} \cdot x} e^{i \frac{t}{2}\left|\xi_{n}^{j}\right|^{2}}\right) \\
& =-\sum_{j=1}^{l}\left(\left|V_{j}\right|^{p-1} V_{j}\right)\left(t, x-t \xi_{n}^{j}\right) e^{i \xi_{n}^{j} \cdot x} e^{i \frac{t}{2}\left|\xi_{n}^{j}\right|^{2}} .
\end{aligned}
$$

We also let

$$
\widetilde{e}_{n}^{l}:=\left(i \partial_{t}+\Delta\right) \widetilde{u}_{n}^{l}+\left|\widetilde{u}_{n}^{l}\right|^{p-1} \widetilde{u}_{n}^{l} .
$$

We shall choose $A>0$ independent of $l$ and $n$ and show, for all $l \gg 1$, there exists $n_{1}=n_{1}(l)$ such that

$$
\left\|\widetilde{u}_{n}^{l}\right\|_{L^{\rho}\left((0, \infty), L^{\gamma}\right)} \leqslant A
$$

holds true for $n \geqslant n_{1}(l)$. By Proposition 2.4, $V_{j}(0)=\psi^{j} \in S_{+}$implies $\left\|V_{j}\right\|_{L^{\rho}\left((0, \infty), L^{\gamma}\right)}<\infty$. Let $\varepsilon>0$ to be chosen later. In light of (6.8) and (6.9), there exists $j_{1}>0$ such that

$$
\left(\sum_{j=j_{1}+1}^{\infty}\left\|\psi^{j}\right\|_{\mathcal{F} H^{1}}^{2}\right)^{1 / 2} \leqslant \varepsilon
$$

Let $\widetilde{v}_{n}^{l}=\widetilde{u}_{n}^{l}-\widetilde{u}_{n}^{j_{1}}$ for $l>j_{1}$. By ([6.6), it hold for any fixed $l>j_{1}$ that

$$
\begin{aligned}
\left\|\widetilde{v}_{n}^{l}(0)\right\|_{\mathcal{F} H^{1}}^{2} & =\left\|\sum_{j=j_{1}+1}^{l} \psi^{j} e^{i \xi_{n}^{j} \cdot x}\right\|_{L^{2}}^{2}+\left\|x \sum_{j=j_{1}+1}^{l} \psi^{j} e^{i \xi_{n}^{j} \cdot x}\right\|_{L^{2}}^{2} \\
& \rightarrow \sum_{j=j_{1}+1}^{l}\left\|\psi^{j}\right\|_{\mathcal{F} H^{1}}^{2} \leqslant \varepsilon^{2}
\end{aligned}
$$

as $n \rightarrow \infty$. Hence, for each $l>j_{1}$, there exists $n_{1,1}=n_{1,1}(l)$ such that

$$
\left\|\widetilde{v}_{n}^{l}(0)\right\|_{\mathcal{F} H^{1}} \leqslant 2 \varepsilon
$$

for all $n>n_{1,1}$. Here, $\widetilde{v}_{n}^{l}$ solves

$$
\widetilde{v}_{n}^{l}(t)=e^{i t \Delta} \widetilde{v}_{n}^{l}(0)+i \int_{0}^{t} e^{i(t-s) \Delta}\left(\left|\widetilde{v}_{n}^{l}\right|^{p-1} \widetilde{v}_{n}^{l}-\widetilde{f}_{n}^{l}\right)(s) d s,
$$

where

$$
\widetilde{f}_{n}^{l}:=\left|\widetilde{v}_{n}^{l}\right|^{p-1} \widetilde{v}_{n}^{l}-\sum_{j=j_{1}+1}^{l}\left(\left|V_{j}\right|^{p-1} V_{j}\right)\left(t, x-t \xi_{n}^{j}\right) e^{i \xi_{n}^{j} \cdot x} e^{i \frac{t}{2}\left|\xi_{n}^{j}\right|^{2}} .
$$


We apply non-admissible Strichartz' estimate (2.4) to (6.12) to yield

$$
\begin{aligned}
\left\|\widetilde{v}_{n}^{l}\right\|_{L^{\rho}\left((0, \infty), L^{\gamma}\right)} \leqslant & \left\|e^{i t \Delta \widetilde{v}_{n}^{l}(0)}\right\|_{L^{\rho}\left((0, \infty), L^{\gamma}\right)}+C_{1}\left\|\widetilde{v}_{n}\right\|_{L^{\rho}\left((0, \infty), L^{\gamma}\right)}^{p} \\
& +C_{1}\left\|\tilde{f}_{n}^{l}\right\|_{L^{\tilde{\rho}^{\prime}\left((0, \infty), \tilde{\gamma}^{\prime}\right)}}
\end{aligned}
$$

where the constant $C_{1}$ depends only on $N$ and $p$. By Lemma 2.1] and (6.11), we have

$$
\left\|e^{i t \Delta} \widetilde{v}_{n}^{l}(0)\right\|_{L^{\rho}\left((0, \infty), L^{\gamma}\right)} \leqslant C \ell\left(\widetilde{v}_{n}^{l}(0)\right) \leqslant C_{2} \varepsilon
$$

where $C_{2}$ depends only on $N$ and $p$. We next estimate $\widetilde{f}_{n}^{l}$. For almost all $t>0, \widetilde{v}_{n}^{l}(t)$ and $V_{l}(t)$ belong to $L^{\gamma}\left(\mathbb{R}^{N}\right)$ for all $n$ and $l$. Fix such $t$. For any fixed $\kappa>0$, there exist compact sets $\Omega^{j} \subset \mathbb{R}^{N}(j \geqslant 1)$ such that $\left\|V_{j}(t)\right\|_{L^{\gamma\left(\left(\Omega^{j}\right)^{c}\right)}} \leqslant \kappa$. Let

$$
\Omega_{n}^{l}:=\bigcup_{j=j_{1}+1}^{l}\left(\Omega^{j}-t \xi_{n}^{j}\right) .
$$

We write $\widetilde{f}_{n}^{l}=F_{1}+F_{2}$ with

$$
\begin{gathered}
F_{1}=\left|\widetilde{v}_{n}^{l}\right|^{p-1} \widetilde{v}_{n}^{l}-\mathbf{1}_{\Omega_{n}^{l}}\left|\widetilde{v}_{n}^{l}\right|^{p-1} \widetilde{v}_{n}^{l}, \\
F_{2}=\mathbf{1}_{\Omega_{n}^{l}}\left|\widetilde{v}_{n}^{l}\right|^{p-1} \widetilde{v}_{n}^{l}-\sum_{j=j_{1}+1}^{l}\left(\left|V_{j}\right|^{p-1} V_{j}\right)\left(t, x-t \xi_{n}^{j}\right) e^{i \xi_{n}^{j} \cdot x} e^{i \frac{t}{2}\left|\xi_{n}^{j}\right|^{2}} .
\end{gathered}
$$

Since

$$
\begin{aligned}
\left\|\widetilde{v}_{n}^{l}\right\|_{L^{\gamma}\left(\left(\Omega_{n}^{l}\right)^{c}\right)} & =\left\|\sum_{j=j_{1}+1}^{l} V_{j}\left(t, x-t \xi_{n}^{j}\right) e^{i \xi_{n}^{j} \cdot x} e^{i \frac{t}{2}\left|\xi_{n}^{j}\right|^{2}}\right\|_{L^{\gamma}\left(\left(\Omega_{n}^{l}\right)^{c}\right)} \\
& \leqslant \sum_{j=j_{1}+1}^{l}\left\|V_{j}\left(t, \cdot-t \xi_{n}^{j}\right)\right\|_{L^{\gamma}\left(\left(\Omega_{n}^{l}\right)^{c}\right)} \leqslant\left(l-j_{1}\right) \kappa,
\end{aligned}
$$

we have

$$
\left\|F_{1}\right\|_{L^{\tilde{\gamma}^{\prime}}} \leqslant\left\|\widetilde{v}_{n}\right\|_{L^{\gamma}\left(\left(\Omega_{n}^{l}\right)^{c}\right)}^{p} \leqslant\left(l-j_{1}\right)^{p} \kappa^{p} .
$$

Since $\Omega^{j}$ is compact and since $t>0$, one sees from (6.6) that there exists $n_{4}$ such that if $n \geqslant n_{4}$ then

$$
\left(\Omega^{j}-t \xi_{n}^{j}\right) \cap\left(\Omega^{k}-t \xi_{n}^{k}\right)=\emptyset
$$

for all $j_{1}<j<k \leqslant l$. Therefore, for $n \geqslant n_{4}$,

$$
\begin{aligned}
\mathbf{1}_{\Omega_{n}^{l}}\left|\widetilde{v}_{n}^{l}\right|^{p-1} \widetilde{v}_{n}^{l} & =\sum_{j=j_{1}+l}^{l} \mathbf{1}_{\left(\Omega^{j}-t \xi_{n}^{j}\right)}\left|\widetilde{v}_{n}^{l}\right|^{p-1} \widetilde{v}_{n}^{l} \\
& =\sum_{j=j_{1}+l}^{l}\left|\mathbf{1}_{\left(\Omega^{j}-t \xi_{n}^{j}\right)} \widetilde{v}_{n}^{l}\right|^{p-1} \mathbf{1}_{\left(\Omega^{j}-t \xi_{n}^{j}\right)} \widetilde{v}_{n}^{l} .
\end{aligned}
$$

Let us recall a well known estimate; there exists constant $C>0$ depending only on $p>1$ such that

$$
\left.|| a\right|^{p-1} a-|b|^{p-1} b\left|\leqslant C\left(|a|^{p-1}+|b|^{p-1}\right)\right| a-b \mid
$$


for any $a, b \in \mathbb{C}$. By this estimate,

$$
\begin{aligned}
\left|F_{2}\right| \leqslant C \sum_{j=j_{1}+1}^{l} & \left(\left|\mathbf{1}_{\left(\Omega^{j}-t \xi_{n}^{j}\right)} \widetilde{v}_{n}^{l}\right|^{p-1}+\left|V_{j}\left(t, x-t \xi_{n}^{j}\right)\right|^{p-1}\right) \\
& \times\left|\mathbf{1}_{\left(\Omega^{j}-t \xi_{n}^{j}\right)} \widetilde{v}_{n}^{l}-V_{j}\left(t, x-t \xi_{n}^{j}\right) e^{i \xi_{n}^{j} \cdot x} e^{i \frac{t}{2}\left|\xi_{n}^{j}\right|^{2}}\right| .
\end{aligned}
$$

It holds that

$$
\begin{aligned}
& \left\|\mathbf{1}_{\left(\Omega^{j}-t \xi_{n}^{j}\right)} \widetilde{v}_{n}^{l}-V_{j}\left(t, x-t \xi_{n}^{j}\right) e^{i \xi_{n}^{j} \cdot x} e^{i \frac{t}{2}\left|\xi_{n}^{j}\right|^{2}}\right\|_{L^{\gamma}} \\
& \leqslant\left\|\widetilde{v}_{n}^{l}-V_{j}\left(t, x-t \xi_{n}^{j}\right) e^{i \xi_{n}^{j} \cdot x} e^{i \frac{t}{2}\left|\xi_{n}^{j}\right|^{2}}\right\|_{L^{\gamma}\left(\Omega^{j}-t \xi_{n}^{j}\right)}+\left\|V_{j}(t)\right\|_{L^{\gamma}\left(\left(\Omega^{j}\right)^{c}\right)} \\
& \leqslant \sum_{j_{1}<m \leqslant l, m \neq j}\left\|V_{m}\left(t, x-t \xi_{n}^{m}\right)\right\|_{L^{\gamma}\left(\Omega^{j}-t \xi_{n}^{j}\right)}+\left\|V_{j}(t)\right\|_{L^{\gamma}\left(\left(\Omega^{j}\right)^{c}\right)} \leqslant\left(l-j_{1}\right) \kappa,
\end{aligned}
$$

where we have used the fact that $\Omega^{j}-t \xi_{n}^{j} \subset\left(\Omega^{m}-t \xi_{n}^{m}\right)^{c}$ to prove the last inequality. It then follows that

$$
\left\|F_{2}\right\|_{L^{\gamma^{\prime}}} \leqslant C \sum_{j=j_{1}+1}^{l}\left(\left\|\widetilde{v}_{n}^{l}(t)\right\|_{L^{\gamma}}^{p-1}+\left\|V_{j}(t)\right\|_{L^{\gamma}}^{p-1}\right)\left(l-j_{1}\right) \kappa \leqslant C \kappa .
$$

Hence, we finally obtain $\left\|\widetilde{f}_{n}^{l}\right\|_{L_{\tilde{\gamma}^{\prime}}} \leqslant C\left(\kappa+\kappa^{p}\right)$ for any $n \geqslant n_{4}$. Thus, for almost all $t>0,\left\|\widetilde{f}_{n}^{l}(t)\right\|_{L \widetilde{\gamma}^{\prime}} \rightarrow 0$ as $n \rightarrow \infty$. On the other hand,

$$
\left\|\widetilde{f}_{n}^{l}(t)\right\|_{L^{\gamma^{\prime}}} \leqslant\left\|\widetilde{v}_{n}^{l}(t)\right\|_{L^{\gamma}}^{p}+\sum_{j=j_{1}+1}^{l}\left\|V_{j}(t)\right\|_{L^{\gamma}}^{p} \leqslant C(p, l) \sum_{j=j_{1}+1}^{l}\left\|V_{j}(t)\right\|_{L^{\gamma}}^{p}
$$

is valid for all $n$. Since the right hand side is independent of $n$ and belongs to $L^{\widetilde{\rho}^{\prime}}((0, \infty))$, we see from Lebesgue's convergence theorem that there exists $n_{1,2}$ such that if $n \geqslant n_{1,2}$ then

$$
\left\|\widetilde{f}_{n}^{l}\right\|_{L \widetilde{\rho}^{\prime}\left((0, \infty), L \widetilde{\gamma}^{\prime}\right)} \leqslant \varepsilon .
$$

Plugging (6.14) and (6.15) to (6.13), we obtain

$$
\left\|\widetilde{v}_{n}^{l}\right\|_{L^{\rho}\left((0, \infty), L^{\gamma}\right)} \leqslant C_{3} \varepsilon+C_{1}\left\|\widetilde{v}_{n}^{l}\right\|_{L^{\rho}\left((0, \infty), L^{\gamma}\right)}^{p}
$$

for $n>n_{1}:=\max \left(n_{1,1}, n_{1,2}\right)$, where $C_{3}$ is independent of $l$ and $n$. We choose $\varepsilon=\varepsilon\left(C_{1}, C_{3}\right)$ so small that this inequality gives

$$
\left\|\widetilde{v}_{n}^{l}\right\|_{L^{\rho}\left((0, \infty), L^{\gamma}\right)} \leqslant 2 C_{3} \varepsilon
$$

For such $\varepsilon$, we obtain the estimate

$$
\left\|\widetilde{u}_{n}^{l}\right\|_{L^{\rho}\left((0, \infty), L^{\gamma}\right)} \leqslant \sum_{j=1}^{j_{1}}\left\|V_{j}\right\|_{L^{\rho}\left((0, \infty), L^{\gamma}\right)}+2 C_{3} \varepsilon=: A
$$

for any $l>j_{1}$ and $n>n_{1}(l)$, which is (6.10). 
Let $\varepsilon_{0}(A)$ be a number given by the long-time perturbation theory (Proposition 4.1 (1)). By (6.4) and (6.7), there exists a number $l_{1}$ such that if $l \geqslant l_{1}$ then we can choose $n_{2}(l)$ so that

$$
\left\|e^{i t \Delta}\left(u_{0, n}-\widetilde{u}_{n}^{l}(0)\right)\right\|_{L^{\rho}\left((0, \infty), L^{\gamma}\right)}=\left\|e^{i t \Delta} W_{n}^{l}\right\|_{L^{\rho}\left((0, \infty), L^{\gamma}\right)} \leqslant \varepsilon_{0}(A)
$$

for all $n \geqslant n_{2}(l)$. We fix $l>\max \left(j_{1}, l_{1}\right)$. Arguing as in the proof of (6.15), we see that there exists $n_{3}(l)$ such that

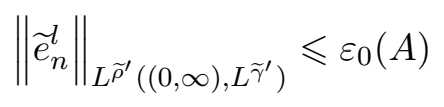

holds for $n \geqslant n_{3}(l)$.

We are now in a position to complete the proof of the claim. Choose $l>$ $\max \left(j_{1}, l_{1}\right)$ and $n \geqslant \max \left(n_{1}(l), n_{2}(l), n_{3}(l)\right)$. Using the long-time perturbation theory, we deduce from (6.10), (6.16), and (6.17) that $\left\|u_{n}\right\|_{L^{\rho}\left((0, \infty), L^{\gamma}\right)} \leqslant$ $c(A)<\infty$, where $u_{n}$ is a solution to (NLS) with $\left.u_{n}\right|_{t=0}=u_{0, n}$. Thanks to Proposition 2.4, this implies $u_{0, n} \in S_{+}$. However, this contradicts with the definition of $u_{0, n}$.

Proof of Theorem 1.3. Choose a sequence $\left\{u_{0, n}\right\}_{n} \subset \mathcal{F} H^{1} \backslash S_{+}$so that $\ell\left(u_{0, n}\right) \leqslant \ell_{c}+\frac{1}{n}$. By scaling, we can assume $\left\|u_{0, n}\right\|_{L^{2}}=1$. We now apply Lemma 6.1. Then, there exist a subsequence of $\left\{u_{0, n}\right\}$, which is denoted again by $\left\{u_{0, n}\right\}$, a function $\psi \in \mathcal{F} H^{1}$ with $\ell(\psi)=\ell_{c}$ and $\|\psi\|_{L^{2}}=1$, and sequences $\left\{W_{n}\right\}_{n} \subset \mathcal{F} H^{1}$ and $\left\{\xi_{n}\right\}_{n} \subset \mathbb{R}^{N}$ such that (6.1) and (6.2) hold.

If $\psi \in S_{+}$then it follows from Proposition 2.4 that $\|V\|_{L^{\rho}\left((0, \infty), L^{\gamma}\right)}<\infty$, where $V$ is a solution of (NLS) with $V(0)=\psi$. Now, apply Proposition 4.1 with $\widetilde{u}(t, x)=V\left(t, x-\xi_{n} t\right) e^{i \xi_{n} \cdot x} e^{i \frac{t}{2}\left|\xi_{n}\right|^{2}}$. Remark that $e \equiv 0$. One also verifies from (6.2) that

$$
\left\|e^{i t \Delta}\left(u_{0, n}-\widetilde{u}(0)\right)\right\|_{L^{\rho}\left((0, \infty), L^{\gamma}\right)} \leqslant\left\|e^{i t \Delta} W_{n}\right\|_{L^{\rho}\left((0, \infty), L^{\gamma}\right)} \rightarrow 0
$$

as $n \rightarrow 0$. Hence, by means of the long-time perturbation theory, we see $u_{0, n} \in S_{+}$for large $n$, which is a contradiction. Thus, $\psi \in \mathcal{F} H^{1} \backslash S_{+}$.

Finally, we prove $\ell_{c}=\inf \left\{\ell(f) \mid f \in \mathcal{F} H^{1} \backslash S\right\}$. For this, it suffices to show

$$
\ell_{c}=\inf _{f \in \mathcal{F} H^{1} \backslash S_{-}} \ell(f)
$$

since

$$
\inf _{f \in \mathcal{F} H^{1} \backslash S} \ell(f)=\min \left(\inf _{f \in \mathcal{F} H^{1} \backslash S_{+}} \ell(f), \inf _{f \in \mathcal{F} H^{1} \backslash S_{-}} \ell(f)\right) .
$$

Let us now recall that if $u(t, x)$ is a solution then $\bar{u}(-t, x)$ is also a solution. This implies $\overline{u_{0}} \in S_{-}$if and only if $u_{0} \in S_{+}$. If $\ell_{c}>\inf \left\{\ell(f) \mid f \in \mathcal{F} H^{1} \backslash S_{-}\right\}$ then there exists $w_{0} \in \mathcal{F} H^{1} \backslash S_{-}$such that $\ell\left(w_{0}\right)<\ell_{c}$. However, it then holds that $\overline{w_{0}} \in \mathcal{F} H^{1} \backslash S_{+}$and $\ell\left(\overline{w_{0}}\right)<\ell_{c}$, which contradicts to the definition of $\ell_{c}$. Hence, $\ell_{c} \leqslant \inf \left\{\ell(f) \mid f \in \mathcal{F} H^{1} \backslash S_{-}\right\}$. A similar argument shows $\ell_{c} \geqslant \inf \left\{\ell(f) \mid f \in \mathcal{F} H^{1} \backslash S_{-}\right\}$. We obtain (6.18). 
Acknowledgments. The author expresses his deep gratitude to Professor Masahito Ohta for fruitful discussions. The author also thanks Professor Masaya Maeda for giving him valuable comments on preliminary version of the manuscript. This research is supported by Japan Society for the Promotion of Science(JSPS) Grant-in-Aid for Young Scientists (B) 24740108.

\section{REFERENCES}

[1] T. Akahori and H. Nawa, Blowup and scattering problems for the nonlinear Schrödinger equations, archived as arXiv1006:1485., 2010.

[2] Hajer Bahouri and Patrick Gérard, High frequency approximation of solutions to critical nonlinear wave equations, Amer. J. Math. 121 (1999), no. 1, 131-175. MR 1705001 (2000i:35123)

[3] Jacqueline E. Barab, Nonexistence of asymptotically free solutions for a nonlinear Schrödinger equation, J. Math. Phys. 25 (1984), no. 11, 3270-3273. MR 761850 (86a:35121)

[4] Thierry Cazenave, Semilinear Schrödinger equations, Courant Lecture Notes in Mathematics, vol. 10, New York University Courant Institute of Mathematical Sciences, New York, 2003. MR 2002047 (2004j:35266)

[5] Thierry Cazenave and Fred B. Weissler, Rapidly decaying solutions of the nonlinear Schrödinger equation, Comm. Math. Phys. 147 (1992), no. 1, 75-100. MR 1171761 (93d:35150)

[6] B. Dodson, Global well-posedness and scattering for the mass critical nonlinear Schrödinger equation with mass below the mass of the ground state, archived as arXiv1104:1114., 2011.

[7] Thomas Duyckaerts, Justin Holmer, and Svetlana Roudenko, Scattering for the nonradial 3D cubic nonlinear Schrödinger equation, Math. Res. Lett. 15 (2008), no. 6, 1233-1250. MR 2470397 (2010e:35257)

[8] DaoYuan Fang, Jian Xie, and Thierry Cazenave, Scattering for the focusing energysubcritical nonlinear Schrödinger equation, Sci. China Math. 54 (2011), no. 10, 20372062. MR 2838120

[9] Damiano Foschi, Inhomogeneous Strichartz estimates, J. Hyperbolic Differ. Equ. 2 (2005), no. 1, 1-24. MR 2134950 (2006a:35043)

[10] J. Ginibre, T. Ozawa, and G. Velo, On the existence of the wave operators for a class of nonlinear Schrödinger equations, Ann. Inst. H. Poincaré Phys. Théor. 60 (1994), no. 2, 211-239. MR 1270296 (95c:35232)

[11] R. T. Glassey, On the blowing up of solutions to the Cauchy problem for nonlinear Schrödinger equations, J. Math. Phys. 18 (1977), no. 9, 1794-1797. MR 0460850 (57 \#842)

[12] Kunio Hidano, Nonlinear Schrödinger equations with radially symmetric data of critical regularity, Funkcial. Ekvac. 51 (2008), no. 1, 135-147. MR 2428826 (2010b:35433)

[13] Justin Holmer and Svetlana Roudenko, A sharp condition for scattering of the radial $3 D$ cubic nonlinear Schrödinger equation, Comm. Math. Phys. 282 (2008), no. 2, 435-467. MR 2421484 (2009h:35403)

[14] Tosio Kato, An $L^{q, r}$-theory for nonlinear Schrödinger equations, Spectral and scattering theory and applications, Adv. Stud. Pure Math., vol. 23, Math. Soc. Japan, Tokyo, 1994, pp. 223-238. MR 1275405 (95i:35276)

[15] Carlos E. Kenig and Frank Merle, Global well-posedness, scattering and blow-up for the energy-critical, focusing, non-linear Schrödinger equation in the radial case, Invent. Math. 166 (2006), no. 3, 645-675. MR 2257393 (2007g:35232)

[16] Sahbi Keraani, On the defect of compactness for the Strichartz estimates of the Schrödinger equations, J. Differential Equations 175 (2001), no. 2, 353-392. MR 1855973 (2002j:35281)

[17] Rowan Killip and Monica Visan, The focusing energy-critical nonlinear Schrödinger equation in dimensions five and higher, Amer. J. Math. 132 (2010), no. 2, 361-424. MR 2654778 (2011e:35357) 
[18] Youngwoo Koh, Improved inhomogeneous Strichartz estimates for the Schrödinger equation, J. Math. Anal. Appl. 373 (2011), no. 1, 147-160. MR 2684466 (2011j:35224)

[19] Kenji Nakanishi and Tohru Ozawa, Remarks on scattering for nonlinear Schrödinger equations, NoDEA Nonlinear Differential Equations Appl. 9 (2002), no. 1, 45-68. MR 1891695 (2003a:35177)

[20] W. A. Strauss, Nonlinear scattering theory, Scattering Theory in Mathematical Physics, J. A. Lavita and J-P. Marchand, eds. Reidel, Dordrecht, Holland-Boston (1974), 53-78.

[21] Yoshio Tsutsumi, Scattering problem for nonlinear Schrödinger equations, Ann. Inst. H. Poincaré Phys. Théor. 43 (1985), no. 3, 321-347. MR 824843 (87g:35221)

[22] — $L^{2}$-solutions for nonlinear Schrödinger equations and nonlinear groups, Funkcial. Ekvac. 30 (1987), no. 1, 115-125. MR 915266 (89c:35143)

[23] M. C. Vilela, Inhomogeneous Strichartz estimates for the Schrödinger equation, Trans. Amer. Math. Soc. 359 (2007), no. 5, 2123-2136 (electronic). MR 2276614 (2008a:35226) 